\title{
On the Nature of Long-Period Dwarf Novae with Rare and Low-Amplitude Outbursts
}

\author{
Mariko KImURA ${ }^{1, *}$, Taichi KATO ${ }^{1}$, Hiroyuki MAeharA ${ }^{2,3}$, Ryoko IshIOKA ${ }^{4,5}$, \\ Berto Monard ${ }^{6,7}$, Kazuhiro NaKaJima ${ }^{8}$, Geoff Stone ${ }^{9}$, Elena P. \\ Pavlenko $^{10}$, Oksana I. AntonyuK ${ }^{10}$, Nikolai V. PIT ${ }^{10}$, Aleksei A. \\ SosnovskiJ ${ }^{10}$, Natalia KATYSheva ${ }^{11}$, Michael Rıchmond ${ }^{12}$, Raúl MıCHEL ${ }^{13}$, \\ Katsura Matsumoto ${ }^{14}$, Naoto KoJiguchi ${ }^{14}$, Yuki Sugiura ${ }^{14}$, Shihei Tei ${ }^{14}$, \\ Kenta Yamamura ${ }^{14}$, Lewis M. CoOK ${ }^{15}$, Richard SABO ${ }^{16}$, lan MILLER ${ }^{17}$, \\ William GofF $^{18}$, Seiichiro KıYota ${ }^{19}$, Sergey Yu. Shugarov ${ }^{11,20}$, Polina \\ Golysheva $^{11}$, Olga Vozyakova ${ }^{11}$, Stephen M. BRInCAT ${ }^{21}$, Hiroshi ITOH ${ }^{22}$, \\ Tamás ToRdal ${ }^{23}$, Colin LITTLEFIELD ${ }^{24}$, Roger D. PICKARD ${ }^{25,26}$, Kenji \\ TANABE $^{27}$, Kenzo KInUGASA ${ }^{28,29}$, Satoshi Honda ${ }^{28,30}$, Hikaru TAGUCH $^{28}$, \\ Osamu HASHIMOTO ${ }^{28}$, and Daisaku NoGAMI ${ }^{1}$ \\ ${ }^{1}$ Department of Astronomy, Graduate School of Science, Kyoto University, Oiwakecho, \\ Kitashirakawa, Sakyo-ku, Kyoto 606-8502 \\ ${ }^{2}$ Okayama Astrophysical Observatory, National Astronomical Observatory of Japan, 3037-5 \\ Honjo, Kamogata, Asakuchi, Okayama, 719-0232 \\ ${ }^{3}$ Okayama Observatory, Graduate School of Science, Kyoto University, 3037-5 Honjo, \\ Kamogata, Asakuchi, Okayama 719-0232 \\ ${ }^{4}$ Institute of Astronomy and Astrophysics, Academia Sinica, 11F of Astronomy-Mathematics \\ Building, AS/NTU No. 1, Section 4, Roosevelt Road, Taipei 10617, Taiwan \\ ${ }^{5}$ Subaru Telescope, National Astronomical Observatory of Japan, 650 North A'ohoku Place, \\ Hilo, HI 96720, USA \\ ${ }^{6}$ Bronberg Observatory, Center for Backyard Astrophysics Pretoria, PO Box 11426, \\ Tiegerpoort 0056, South Africa \\ ${ }^{7}$ Kleinkaroo Observatory, Center for Backyard Astrophysics Kleinkaroo, Sint Helena 1B, PO \\ Box 281, Calitzdorp 6660, South Africa \\ ${ }^{8}$ Variable Star Observers League in Japan (VSOLJ), 124 Teradani, Isato-cho, Kumano, Mie \\ 519-4673 \\ ${ }^{9}$ American Association of Variable Star Observers, 49 Bay State Rd., Cambridge, MA 02138, \\ USA \\ ${ }^{10}$ Crimean Astrophysical Observatory, 298409, Nauchny, Republic of Crimea \\ ${ }^{11}$ Sternberg Astronomical Institute, Lomonosov Moscow State University, Universitetsky Ave., \\ 13, Moscow 119992, Russia \\ ${ }^{12}$ Physics Department, Rochester Institute of Technology, Rochester, New York 14623, USA \\ ${ }^{13}$ Instituto de Astronomía UNAM, Apartado Postal 877, 22800 Ensenada B.C., México \\ ${ }^{14}$ Osaka Kyoiku University, 4-698-1 Asahigaoka, Kashiwara, Osaka 582-8582 \\ ${ }^{15}$ Center for Backyard Astrophysics Concord, 1730 Helix Ct. Concord, California 94518, USA \\ ${ }^{16} 2336$ Trailcrest Dr., Bozeman, Montana 59718, USA \\ ${ }^{17}$ Furzehill House, Ilston, Swansea, SA2 7LE, UK \\ ${ }^{18}$ American Association of Variable Star Observers (AAVSO), 13508 Monitor Lane, Sutter \\ Creek, California 95685, USA
}


${ }^{19}$ Variable Star Observers League in Japan (VSOLJ), 7-1 Kitahatsutomi, Kamagaya, Chiba 273-0126

${ }^{20}$ Astronomical Institute of the Slovak Academy of Sciences, 05960 Tatranska Lomnica, Slovakia

${ }^{21}$ Flarestar Observatory, San Gwann SGN 3160, Malta

${ }^{22}$ Variable Star Observers League in Japan (VSOLJ), 1001-105 Nishiterakata, Hachioji, Tokyo 192-0153

${ }^{23}$ Polaris Observatory, Hungarian Astronomical Association, Laborc utca 2/c, 1037 Budapest, Hungary

${ }^{24}$ Astronomy Department, Wesleyan University, Middletown, Connecticut 06459, USA

${ }^{25}$ The British Astronomical Association, Variable Star Section (BAA VSS), Burlington House, Piccadilly, London, W1J ODU, UK

${ }^{26} 3$ The Birches, Shobdon, Leominster, Herefordshire, HR6 9NG, UK

${ }^{27}$ Department of Biosphere-Geosphere System Science, Faculty of Informatics, Okayama University of Science, 1-1 Ridai-cho, Okayama, Okayama 700-0005

${ }^{28}$ Gunma Astronomical Observatory, 6860-86 Nakayama, Takayama, Agatsuma, Gunma 377-0702

${ }^{29}$ Nobeyama Radio Observatory, National Astronomical Observatory of Japan, National Institutes of Natural Sciences, 462-2 Nobeyama, Minamimaki, Minamisaku, Nagano 384-1305

${ }^{30}$ Nishi-Harima Astronomical Observatory, Center for Astronomy, University of Hyogo, 407-2 Nishigaichi, Sayo-cho, Sayo, Hyogo 679-5313

*E-mail: mkimura@kusastro.kyoto-u.ac.jp

Received; Accepted

\section{Abstract}

There are several peculiar long-period dwarf-nova like objects, which show rare, low-amplitude outbursts with highly ionized emission lines. 1SWASP J162117+441254, BD Pav, and V364 Lib belong to this kind of objects. Some researchers even doubt whether 1SWASP J1621 and V364 Lib have the same nature as normal dwarf novae. We studied the peculiar outbursts in these three objects via our optical photometry and spectroscopy, and performed numerical modeling of their orbital variations to investigate their properties. We found that their outbursts lasted for a long interval (a few tens of days), and that slow rises in brightness were commonly observed during the early stage of their outbursts. Our analyses and numerical modeling suggest that 1SWASP J1621 has a very high inclination, close to $90 \mathrm{deg}$, plus a faint hot spot. Although BD Pav seems to have a slightly lower inclination ( $75 \mathrm{deg})$, the other properties are similar to those in 1SWASP J1621. On the other hand, V364 Lib appears to have a massive white dwarf, a hot companion star, and a low inclination ( $35 \mathrm{deg}$ ). In addition, these three objects possibly have low transfer rate and/or large disks originating from the long orbital periods. We find that these properties of the three objects can explain their infrequent and low-amplitude outbursts within the context of the disk instability model in normal dwarf novae without strong magnetic field. In addition, we suggest that the highly-ionized emission lines in outburst are observed due to a high inclination and/or a massive white dwarf. More instances of this class of object may be unrecognized, since their unremarkable outbursts can be easily overlooked.

Key words: accretion, accretion disks - novae, cataclysmic variables - stars: dwarf novae - stars: individual (1SWASP J162117+441254, BD Pavonis, V364 Libra) 


\section{Introduction}

Statistical studies on dwarf novae show many dwarf novae having long orbital periods (more than 3 hours) go through outbursts with typical amplitudes of 2-5 mag, and that the intervals between their outbursts are usually less than 1 year (see Warner 1995 for a review). It is widely accepted that dwarf novae showing low outburst amplitudes undergo outbursts more frequently (Warner 1987). A very low-amplitude and rare outburst was, however, discovered for the first time in a dwarf-nova like object, ASAS 150946-2147.7 = V364 Lib, (Pojmanski et al. 2009). This object has a long orbital period of 16.86 hours, and enters its outbursts with amplitudes of $\sim 1$ mag every few years (Wils 2011; vsnet-alert 14271; vsnet-alert 20877). In addition, highly ionized emission lines (He II 4686 and C III/N III) were detected in the 2009 outburst. Although this led Kinugasa et al. (2009) to propose that this system is a black-hole binary, Uemura et al. (2009) reported that the X-ray luminosity during the outburst was comparable to the quiescent X-ray luminosity of black-hole binaries. Thus this object contradicts the general rule of dwarf novae, and the origin of the peculiar outburst and highly ionized emission lines remains a mystery.

On June 3.45, 2016 (UT), a small-amplitude and very rare outburst was discovered in another object by Catalina RealTime Transient Survey (CRTS) (Drake et al. 2016; Drake et al. 2014a). Its name is 1SWASP J162117+441254 (hereafter 1SWASP J1621), and this object also has a long orbital period of 4.99 hours (Lohr et al. 2013a). The outburst amplitude was $\sim 2$ mag and only one outburst was detected over ten years before the 2016 outburst in this system (Kjurkchieva et al. 2017). The strong He II 4686 emission line observed in the outburst was similar to that in the 2009 outburst of V364 Lib (Scaringi et al. 2016). Moreover, this object had been previously identified as a W UMa-type system on the basis of SDSS colors and double-waved orbital variations in quiescence (Palaversa et al. 2013; Lohr et al. 2013b; Drake et al. 2014b); hence, the 2016 outburst was expected to be the onset of a merger of two mainsequence (MS) stars, which was reminiscent of the 2008 eruption in V1309 Sco. Scaringi et al. (2016), however, confirmed by their spectroscopic observations that this outburst occurred in an accreting compact binary rather than a MS+MS merger. In addition, this system showed much deeper eclipses in outburst than in quiescence (Zejda, Pejcha 2016; Kjurkchieva et al. 2017; Zola et al. 2017; Sec. 3.1 in this paper). This phenomenon made us notice the similarity between this object and the almost forgotten dwarf nova BD Pav, which has an orbital period of 4.3 hours (Barwig, Schoembs 1983). BD Pav has also shown relatively low-amplitude and rare outbursts, and the He II 4686 emission line was observed in its 1985 outburst. The emission line was not clearly double-peaked, in spite of the deep eclipses in outburst suggesting its high inclination (Barwig, Schoembs 1987).
Recently, the two objects GY Hya and V1129 Cen, which showed outbursts, spectra, and orbital variability similar to those in 1SWASP J1621, BD Pav and V364 Lib, have been studied (Bruch, Monard 2017; Bruch 2017). In addition, HS 0218+3229 (hereafter HS 0218) shows rare outbursts and has a long orbital period, although the outburst amplitude is not so small, 4 mag in the $V$ band (Golysheva et al. 2012; Golysheva et al. 2013; Katysheva et al. 2015), and it was pointed out that this object is similar to 1SWASP J1621 (Katysheva et al. 2017). Thus this kind of object recently has started to attract attention, but there is not yet any coherent explanation to produce its peculiar outburst and spectral behavior. Moreover, some of these objects were not clearly identified with normal dwarf novae, and it is even doubted whether the disk instability model, which is believed to be the most plausible model of outbursts in non-magnetic dwarf novae (Osaki 1996 for a review), can explain the outbursts in this kind of object. For example, Dr. Breedt claims that 1SWASP J1621 has a hot companion star (Waagen 2017). Although this assumption would be true in V1129 Cen according to Bruch (2017), it is not consistent with the spectroscopic observations of 1SWASP J1621 and BD Pav (Thorstensen 2016; Sion et al. 2008; Friend et al. 1990). In addition, Qian et al. (2017) even argued that the eruption in 1SWASP J1621 was not a dwarf-nova outburst. They considered the accretion disk is barely formed due to the strong magnetic activity of the companion star in this system, and that a sudden increase of mass transfer from the companion causes the eruption. We therefore aimed to investigate the nature of 1SWASP J1621, BD Pav, and V364 Lib, and to examine by analyzing our photometric and spectroscopic observational data and modeling their orbital variations, whether some special feature which is not presented in normal dwarf novae is necessary to explain their peculiar outbursts.

The plan of this paper is as follows: the data reduction and techniques of analyses are described in Sec. 2. We present our optical photometry of 1SWASP J1621, BD Pav, and V364 Lib in Sec. 3, and optical spectroscopy of V364 Lib in Sec. 4. In addition, we perform numerical modeling to explain the orbital variations in Sec. 5. In Sec. 6, we discuss our results, and in Sec. 7, our conclusions are summarized.

\section{Observation and Analysis}

\subsection{Photometry}

Time-resolved CCD photometry was carried out by the VSNET collaboration team. The telescopes and sites are summarized in Table E1. Tables E2, E3, E4 show the logs of photometric observations of 1SWASP J1621, BD Pav, and V364 Lib, respectively. We also used the data downloaded from the AAVSO archive $^{1}$, the ASAS-3 data archive (Pojmanski, Maciejewski

\footnotetext{
${ }^{1}<$ http://www.aavso.org/data/download/>
} 
2004) and the ASAS-SN data archive (Davis et al. 2015). All of the observation times were converted to barycentric Julian date (BJD). Before making the analyses, we applied zero-point corrections to each observer by adding constants as for 1SWASP J1621 and V364 Lib. We did not do that as for BD Pav, since all of the observations were performed by one observer. The magnitude scales of each site were adjusted to that of the Crimean Observatory system (CRI in Table E2), where TYC

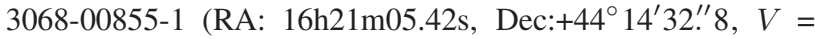
12.2) was used as the comparison star for 1SWASP J1621. The constancy of the comparison star was checked by nearby stars in the same images. The magnitude of the comparison star was measured by the AAVSO Photometric All-Sky Survey (APASS: Henden et al. 2016) from the AAVSO Variable Star Database. ${ }^{2}$ As for V364 Lib, we adjusted the magnitude of our observational data to that of the ASAS data, assuming the magnitude in a clear filter is almost the same as that in the $V$ band. The exposure times of the observations by the VSNET team were 30-300 s as for 1SWASP J1621 and V364 Lib, and $30 \mathrm{~s}$ as for BD Pav.

\subsection{Period Analyses}

We used the phase dispersion minimization (PDM) method (Stellingwerf 1978) for period analyses. We subtracted the global trend of the light curve by locally weighted polynomial regression (LOWESS: Cleveland 1979) before performing the PDM analysis. The $1 \sigma$ error of the best estimated period by the PDM analysis was determined by the methods in Fernie (1989) and Kato et al. (2010). A variety of bootstraps was used for estimating the robustness of the result of PDM. We analyzed about 100 samples which randomly contain $50 \%$ of observations, and performed a PDM analysis for these samples.

\subsection{Spectroscopy}

Our spectroscopic observations of V364 Lib were carried out by using the Gunma Astronomical Observatory’s 1.5-m telescope equipped with Gunma LOW resolution Spectrograph and imager (GLOWS) in the 2009 outburst and using the High Dispersion Spectrograph (HDS: Noguchi et al. 2002) attached to the 8.2-m Subaru telescope of National Astronomical Observatory of Japan (NAOJ) in quiescence just after the outburst. The spectral coverage of the two spectrographs was about 4000-8000 and 3000-10000 $\AA$, respectively, and the spectroscopic resolutions $(R=\lambda / \Delta \lambda)$ are 400-500 for GLOWS and 90,000 for HDS. Data reduction was conducted using IRAF $^{3}$ in the standard manner (bias subtraction, flat fielding, aperture

\footnotetext{
${ }^{2}<$ http://www.aavso.org/vsp >

${ }^{3}$ IRAF is distributed by the National Optical Astronomy Observatories, which is operated by the Association of Universities for Research in Astronomy, Inc., under cooperate agreement with the National Science Foundation.
}

determination, scattered light subtraction, spectral extraction, wavelength calibration, normalization by the continuum, and heliocentric radial-velocity correction). We measured the radial velocities of the object by fitting gaussians with the task SPLOT in IRAF. The observation dates, exposure times, and observatories are summarized in Table E5. The estimated radial velocities are given in Tables E6 and E7.

\section{Photometric Light Curves}

\subsection{SWASP J1621}

In the 2016 outburst, the amplitude of its outburst was relatively small, $\sim 2$ mag. The duration of the outburst was probably about two weeks, and the brightness rose slowly in the early stage of the event. The overall optical light curve of the 2016 outburst in 1SWASP J1621 with a clear filter is displayed in figure 1. This system showed deep eclipsing variations on its light curve. As this system was fading, the primary minima became shallower and the secondary minima became deeper. We display in figure 2 the nightly averaged phase profiles in a clear filter during the fading stage in the outburst. This system showed W UMa-type orbital variations with amplitudes of $\sim 0.6$ mag in quiescence and deep primary minima in outburst. The phase-averaged profiles around the outburst maximum and in quiescence during BJD 2457552-2457559 in the $R_{\mathrm{C}}, V$, and $B$ bands are exhibited in figure 3 . The profile around the outburst maximum was derived from the multi-color observational data obtained by Mic during BJD 2457542.8-2457546.8 (see the annotations in Table E2), which include 3 cycles of the eclipses. When folding the outburst light curve, we subtracted the long-term trend by using locally weighted polynomial regression (LOWESS: Cleveland 1979) after removing the part of the phase representing the deep eclipse. The derived outburst amplitude is $\sim 1 \mathrm{mag}$ in the $R_{\mathrm{C}}$ band from the phase-averaged light curves. During the outburst, we observed colors of $V-R \sim 0.4$ mag and $B-V \sim 0.1 \mathrm{mag}$ outside eclipses; in quiescence, those colors were $V-R \sim 0.6$ mag and $B-V \sim 1.0$ mag. In the $B$ band, the flux of the maxima around phase 0.25 was a little lower than that of the maxima around phase 0.75 .

\subsection{BD Pav}

We performed the optical photometry of the 2006 outburst of BD Pav in the past. The outburst amplitude was $\sim 2.5 \mathrm{mag}$. The overall light curve of this outburst is shown in figure 4. In addition, we confirmed the deep primary minima in this outburst similar to those in the 2016 outburst of 1SWASP J1621. In figure 5 , we show the phase-averaged profiles both in the 2006 outburst and in quiescence. In folding the outburst light curve, we subtracted the long-term trend using the same method described for 1SWASP J1621. We can see that this object showed the 


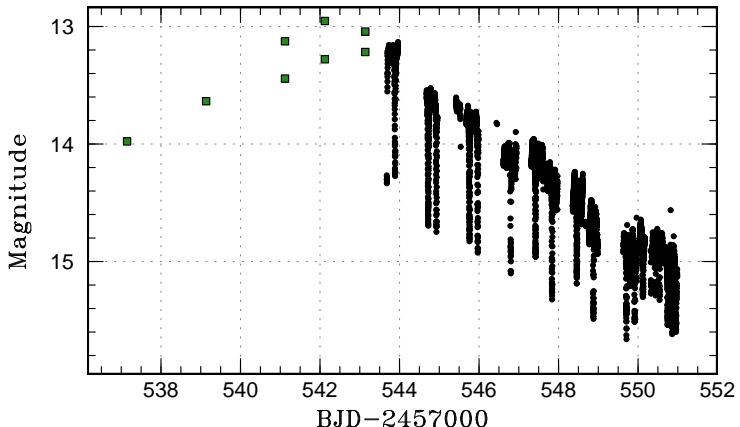

Fig. 1. Overall light curve of the 2016 outburst in 1SWASP J1621 (BJD 2457537-2457551). The filled rectangles represent the snap-shot observations by Hiroyuki Maehara (Maehara 2016).

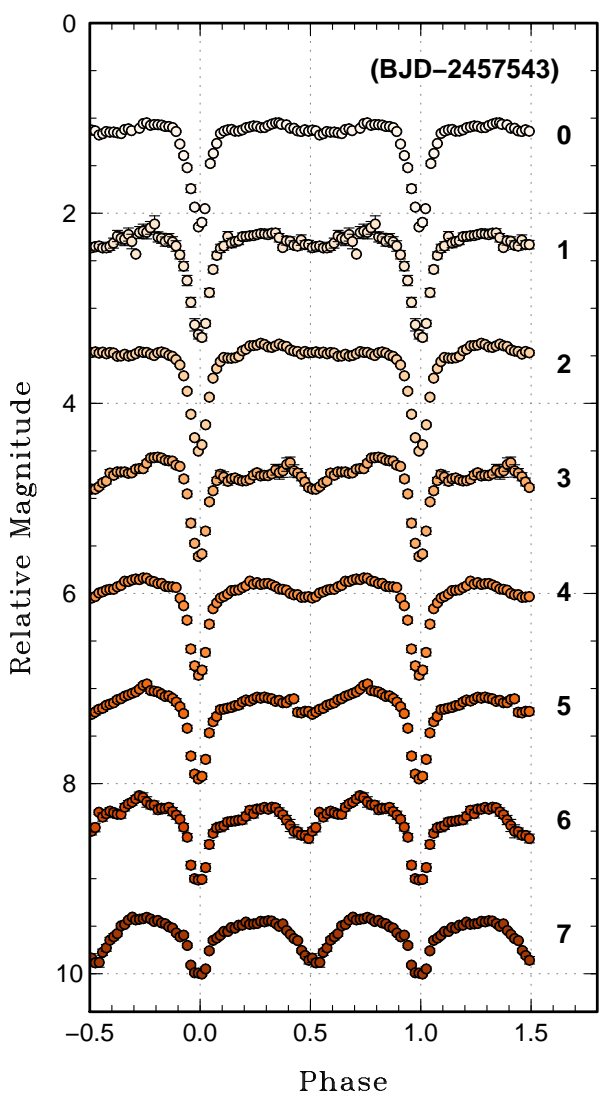

Fig. 2. Nightly eclipsing variations in magnitudes with clear filter in 1SWASP J1621 during the 2016 outburst (BJD 2457543-2457550). The numbers at the right end represent the days from the beginning of the outburst.

much deeper primary minima in the outburst than in quiescence and that double-waved orbital modulations with amplitudes of $\sim 0.3$ mag were observed in quiescence. The main characteristics of these eclipsing profiles are consistent with the results in Figures 2 and 3 in Barwig, Schoembs (1987). Although the rising phase in the 2006 outburst was not observed in our pho-
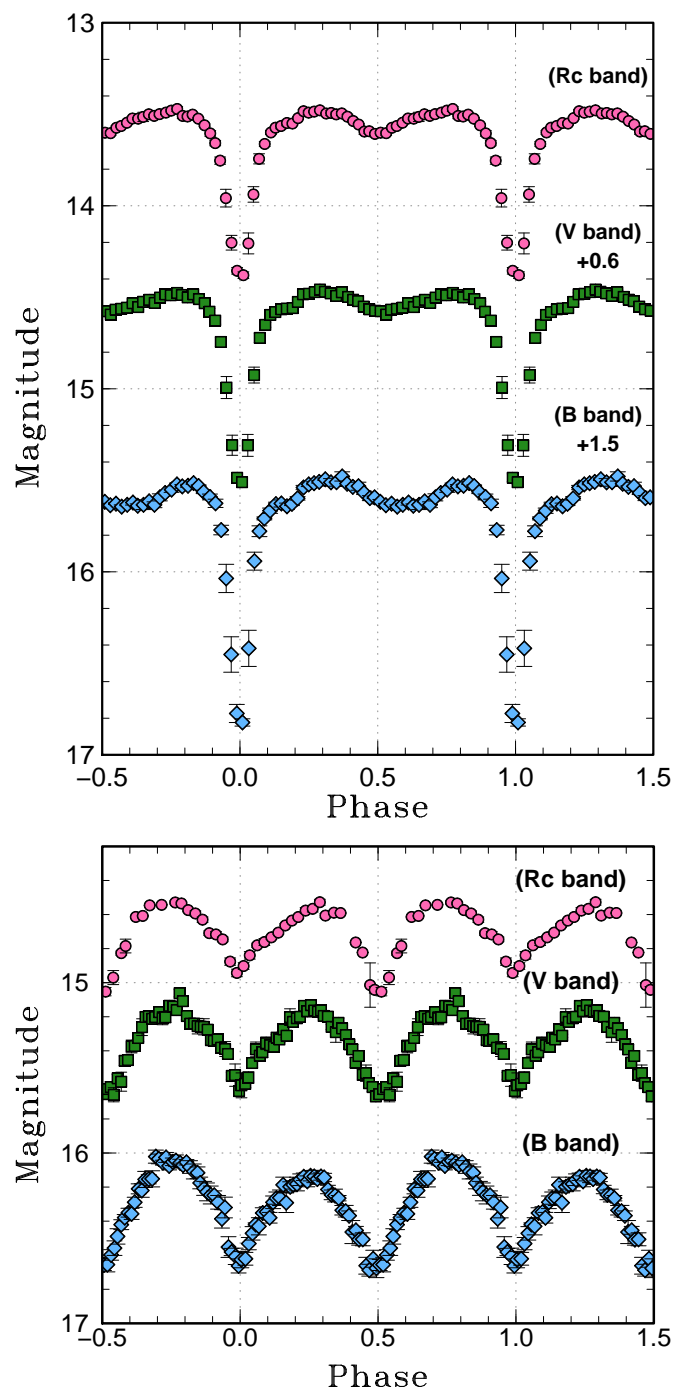

Fig. 3. Phase-averaged light curves of orbital variations in the outburst state during BJD 2457542.8-2457546.8 (the upper panel) and in quiescence (the lower panel) during BJD 2457552-2457559 in 1SWASP J1621. Diamonds, rectangles, and circles represent the $B, V$ and $R_{\mathrm{C}}$-bands phase profiles, respectively. In the upper panel, the $V$-band and $B$-band magnitudes are offset by 0.6 and 1.5 , respectively, for visibility. The folding period is 0.207852 d, which was reported by Drake et al. (2014a). The epochs in the outburst maximum and in quiescence are BJD 2457546.59 and BJD 2457549.7079, respectively.

tometry, many other outbursts in BD Pav showed slow rises. We show measurements in the $V$ band by ASAS during the recent 2015 and 2017 outbursts in figure 6 as examples.

\subsection{V364 Lib}

We carried out the optical photometry of the 2009 outburst of V364 Lib in the past. The duration was about 35 days and the outburst amplitude was $\sim 1$ mag. A slow rise during the early stage of the outburst was observed, as in the outbursts of 


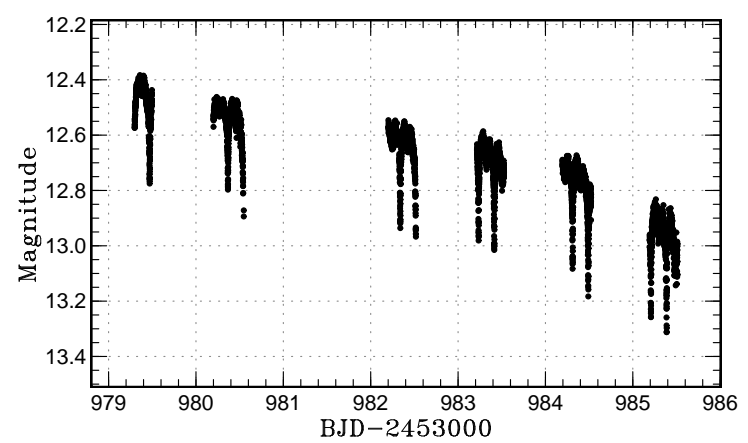

Fig. 4. Overall light curve of the 2006 outburst in BD Pav with a clear filter (BJD 2453979-2453986).
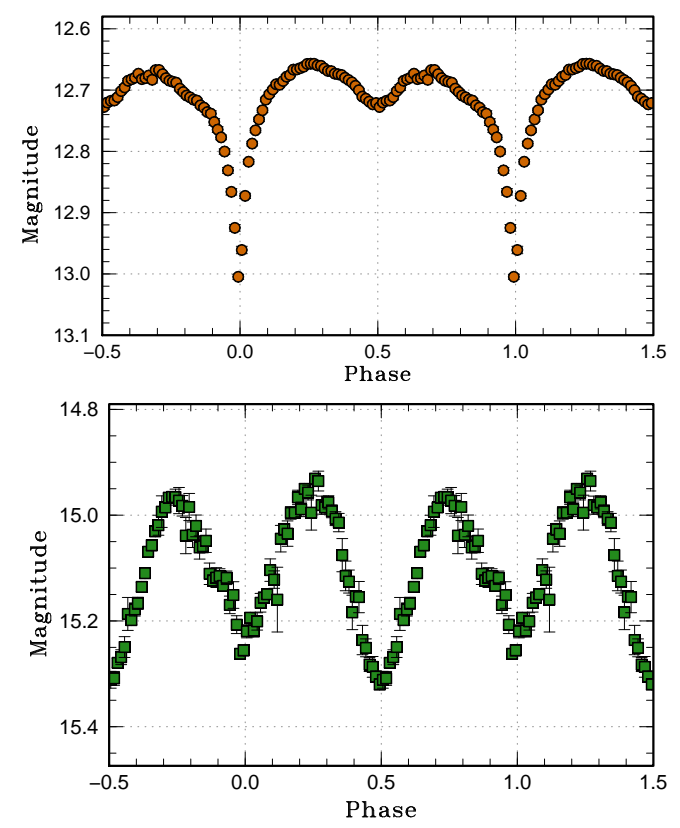

Fig. 5. Phase-averaged profiles of orbital variations of BD Pav in the 2006 outburst during BJD 2453979-2453986 (the upper panel) and in quiescence during BJD 2456454-2456466 (the lower panel). The folding period is 0.17930 , which is reported in Barwig, Schoembs (1983). The epochs are BJD 2453982.339 in the outburst and BJD 2456454.725 in quiescence.

1SWASP J1621 and BD Pav. Its overall light curve is displayed in figure 7. Small-amplitude $(\sim 0.07 \mathrm{mag})$ and double-waved orbital variations were detected in quiescence, as displayed in figure 8 . In outburst, these orbital variations were expected to be observed with an amplitude of $\sim 0.025 \mathrm{mag}$; however they were not detected due to other small-amplitude variations on time scales of $\sim 0.1 \mathrm{~d}$, which are shorter than the orbital period (and below the limits of our precision). A part of the variations in outburst is displayed in figure 9 .

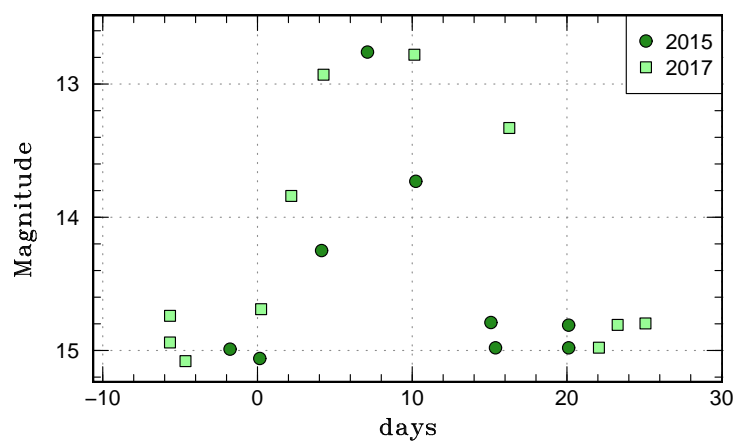

Fig. 6. Monitoring BD Pav in the $V$ band by ASAS during the 2015 and 2017 outbursts. The horizontal axis represents time in days from each outburst. The circles and squares represent the 2015 and 2017 outbursts, respectively.

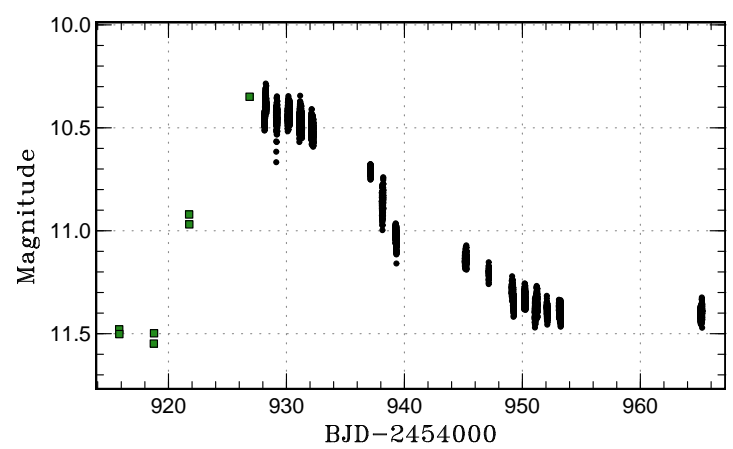

Fig. 7. Overall light curve of the 2009 outburst in V364 Lib with a clear filter (BJD 2454928-2454954). The squares represent observations in the $V$ band by ASAS.

\section{Spectroscopy in V364 Lib}

We detected absorption lines in the Balmer series and strong emission lines of He II 4686 and C III/N III during the early stage of the 2009 outburst in V364 Lib. An example of optical spectra in the early stage of the outburst is given in figure 10

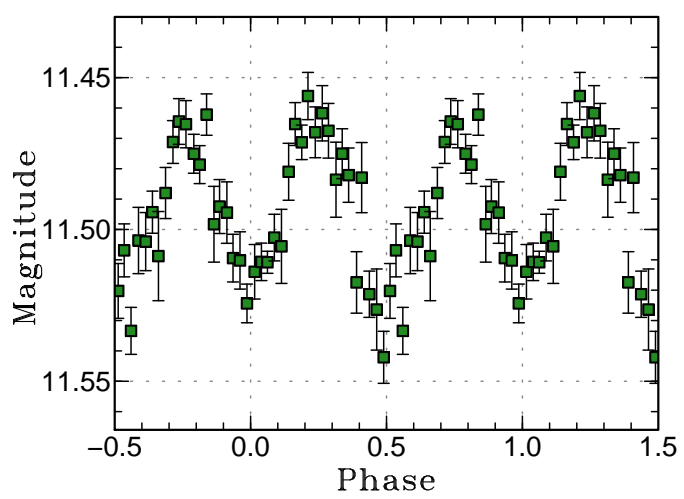

Fig. 8. Phase-averaged profile of orbital variations in quiescence in V364 Lib. The folding orbital period estimated by the PDM method is $0.7024293(1053) d$. The epoch is BJD 2453880.244654. 


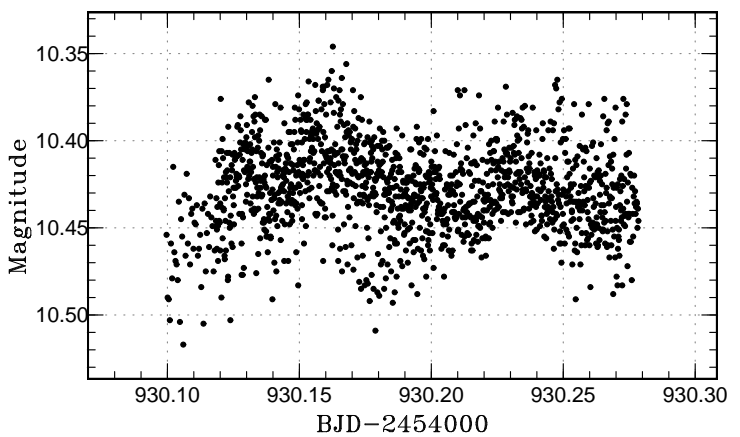

Fig. 9. An example of the variations on timescales shorter than the orbital period in the 2009 outburst in V364 Lib.

with the spectrum in quiescence for comparison. In quiescence, the contribution of the companion star was dominant in the optical spectra. The companion star was determined to be an F-type star with surface temperature between $6,500 \mathrm{~K}$ and $6,750 \mathrm{~K}$ by comparing the observational spectra with the synthetic spectra which are computed with a synthetic stellar atmosphere interpolated from the Castelli, Kurucz (2004) grid ${ }^{4}$. An example of optical spectra in quiescence is given in figure 11 with some synthetic spectra.

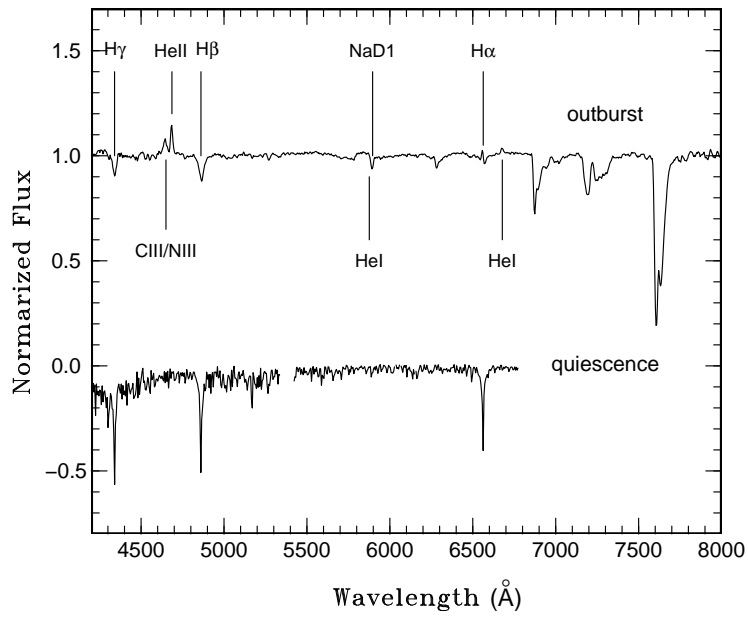

Fig. 10. Spectrum of V364 Lib on April 7th, 2009 in the outburst state. For reference, an example of the spectra in quiescence on May 5th, 2009 is also displayed. For visibility, an offset of -1.0 is added to the quiescent spectrum.

We deduced that the central compact object in this system is a white dwarf as follows. The estimated value of the radial velocity of the companion star (K2), using the average value of $\mathrm{H} \beta$ and $\mathrm{Mg}$ absorption lines in quiescence, was $74.1 \pm 1.3 \mathrm{~km} / \mathrm{s}$ (see figure 12). Although the error was somewhat large, the derived value of the radial velocity of the central compact object (K1) from the He II emission line in the outburst state was

\footnotetext{
${ }^{4}$ https://www.oact.inaf.it/castelli/castelli/grids.html
}

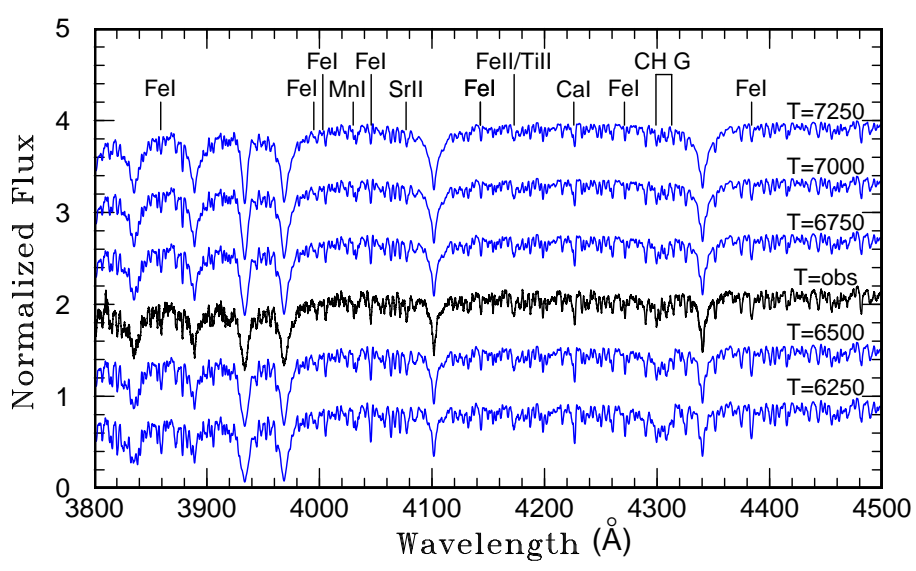

Fig. 11. Spectrum of V364 Lib in quiescence. The black line represents observations on May 5th, 2009. The blue lines represent the broadened synthetic spectra in Castelli, Kurucz (2004).

$107.8 \pm 12.6 \mathrm{~km} / \mathrm{s}$ (see figure 13). We then assumed that the systemic velocity is the same as that in quiescence, and that the orbital period is $0.7024293 \mathrm{~d}$. Thus the mass ratio $\left(q \equiv M_{2} / M_{1}\right)^{5}$ was estimated to be $1.5 \pm 0.2$. The surface temperature of the companion suggests that its mass is close to $1.4 M_{\odot}$ (Allen 1973), and hence, the mass of the compact object was estimated to be $1.0 \pm 0.2 M_{\odot}$ from the mass ratio. This value and the faint X-ray luminosity during outburst (Uemura et al. 2009) indicate that the compact object in this system should be a white dwarf. In addition, the inclination angle was estimated to be $\sim 35 \mathrm{deg}$ from the equation $\sin ^{3} i=P K_{2}{ }^{3}(1+q)^{2} /\left(2 \pi G M_{1}\right)$, using the estimated values of $\mathrm{K} 2$, mass ratio, and mass of the primary, which is consistent with a $v \sin i$ value of $85 \mathrm{~km} / \mathrm{s}$ estimated from the absorption lines in quiescence.

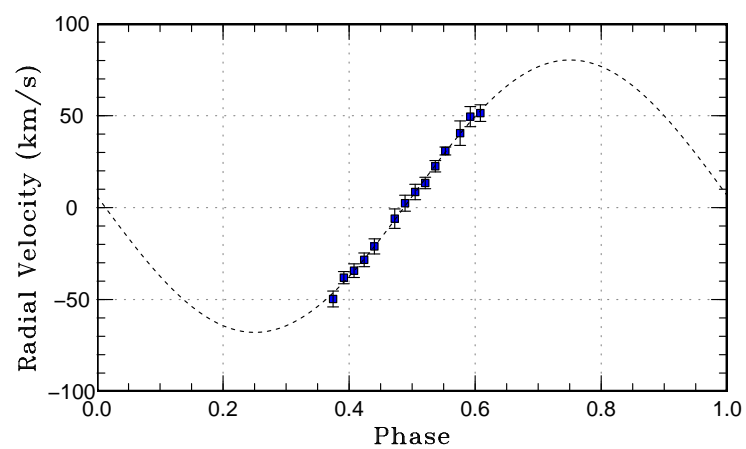

Fig. 12. Radial velocity extracted from the average value of $\mathrm{H} \beta$ and $\mathrm{Mg}$ ab sorption lines in quiescence under the assumption that the orbital period is $0.7024293 \mathrm{~d}$. The rectangles represent the observations. The dashed line is the best fitted sine curve with the semi-amplitude of $74.1 \pm 1.1 \mathrm{~km} / \mathrm{s}$ and the systemic velocity of $6.2 \pm 5.4 \mathrm{~km} / \mathrm{s}$.

\footnotetext{
${ }^{5}$ Here, $M_{1}$ and $M_{2}$ represent the mass of the primary star and that of the secondary star, respectively.
} 


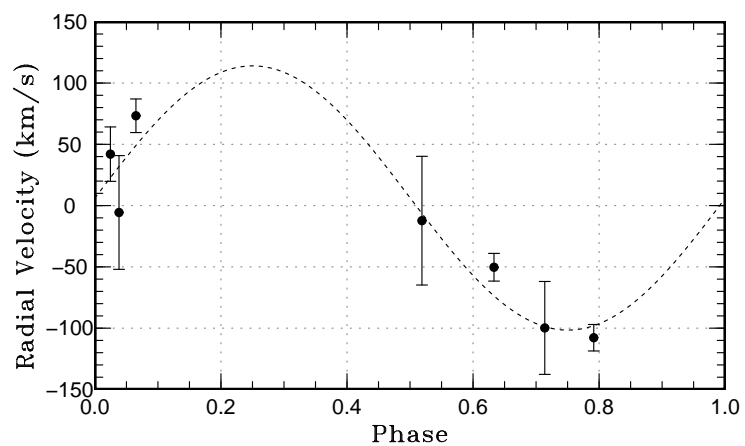

Fig. 13. Radial velocity extracted from He II 4686 emission line in the 2009 outburst under the assumption that the orbital period is $0.7024293 \mathrm{~d}$. The points represent the observations. The dashed line is the best fitted sine curve with the semi-amplitude of $107.8 \pm 12.6 \mathrm{~km} / \mathrm{s}$ and the systemic velocity of $6.2 \mathrm{~km} / \mathrm{s}$

\section{Numerical Modeling of Orbital Variations}

\subsection{Premises}

As described in the introduction, the observational W UMa-type orbital variations in quiescence are sometimes ascribed to their hot companions in 1SWASP J1621 and BD Pav (Waagen 2017; Barwig, Schoembs 1983), but there is evidence that 1SWASP J1621 and BD Pav have cool companion stars (Thorstensen 2016; Sion et al. 2008; Friend et al. 1990). In addition, our spectral analyses in Sec. 4 suggest that V364 Lib has a hot companion star and a low inclination. We thus wondered if the double-waved modulations in quiescence and the outburst amplitudes are explained without hot companions as for 1SWASP J1621 and BD Pav, and with the nature suggested by our results in Sec. 4 in V364 Lib, via the modeling of the orbital phase profiles. Since we have the $R_{\mathrm{C}}, V$, and $B$-band phase profiles in outburst (see figure 3) in 1SWASP J1621, we considered reproducing their characteristics in this object.

Before presenting our modeling, we summarize the information derived from our observations. According to Horne (1985), the width of the eclipse of a white dwarf is determined by the mass ratio and inclination of its system. The width is approximately estimated as the width of eclipse at half depth if the disk is axisymmetric. Regarding the inflection phases in outburst in the $R_{\mathrm{C}}$-band profile for 1SWASP J1621 and in the clear-filter profile for BD Pav as the ends of eclipses, the rough estimates of the width of eclipse of the white dwarf are $\sim 0.10$ in 1SWASP J1621 and $\sim 0.06$ in BD Pav, respectively. Substituting the half values of them and the values of mass ratios to equation (3) in Horne et al. (1982), which represents the relation between the inclination, mass ratio, and width of eclipse of a white dwarf, we obtain inclinations of $~ 87-$ deg in 1SWASP J1621 and 75 -deg in BD Pav, respectively. These values are consistent with those extracted by Kjurkchieva et al. (2017); Zola et al. (2017); Friend et al. (1990); Sion et al. (2008). In addition, the almost symmetric phase-averaged profiles around the primary minima in these two objects seem to imply a dim hot spot (see figures 3 and 5).

\subsection{Method}

\subsubsection{Code}

We used the numerical code which is described in detail by Hachisu, Kato (2001), Hachisu, Kato (2003a), and Hachisu, Kato (2003b). In this code, the companion star fills its Roche lobe and a circular orbit is assumed. The surfaces of the white dwarf, companion star, and accretion disk are divided into patches, and each patch emits photons by black body radiation at the local temperature. The patches of the disk and companion are irradiated by the front-side patches of the white dwarf if there is no patch between them. The non-irradiated surface temperature is determined by viscous heating of the standard disk model (Shakura, Sunyaev 1973). The total luminosity of the binary system is calculated by summing up the luminosity from all visible patches. The surface patch elements are $32 \times 64$ $(\theta \times \phi)$ for the companion, $32 \times 64(\theta \times \phi \times$ up and down side $)$ for the accretion disk, and $16 \times 32$ for the white dwarf.

\subsubsection{Parameters}

Using the code described in Sec. 5.2.1, we computed eclipsing light variations with various test parameters: the inclination angle, temperature distribution, height and radius of the accretion disk. Inclinations were varied over ranges of 84-90 deg, 72-78 deg, and 30-40 deg by steps of 1 deg for 1SWASP J1621 and BD Pav, V364 Lib, respectively, by considering the estimation in Sec. 4 and Sec. 5.1. Tested disk radii were 0.5, 0.6, 0.7, 0.8, $0.9 R_{\mathrm{L} 1}$. Here, $R_{\mathrm{L} 1}$ is defined as $R_{\mathrm{L} 1}=a /\left(1.0015+q^{0.4056}\right)$, where $a$ is the binary separation (equation (2.4c) in Warner 1995).

We gave the temperature distribution of the accretion disk in outburst, postulating that the disk is steady at the outburst maximum (Wood et al. 1986) as follows:

$T=T_{*}\left(\frac{r}{r_{\text {in }}}\right)^{-3 / 4}\left[1-\left(\frac{r_{\text {in }}}{r}\right)^{1 / 2}\right]^{1 / 4}, T_{*}=\left(\frac{3 G M \dot{M}}{8 \pi \sigma r_{\text {in }}^{3}}\right)^{1 / 4}(1)$

Here, $r, r_{\text {in }}, G, M, \dot{M}$ and $\sigma$ represent the distance from the central object to a given ring of the disk, inner disk radius, constant of gravitation, mass of the primary star, mass accretion rate at a given radius, and Stefan-Boltzmann constant, respectively (Shakura, Sunyaev 1973), and $\dot{M}$ is different from the masstransfer rate from a companion star. The value of $r_{\text {in }}$ is set to 0.02 in units of the binary separation. The $\alpha$-value is also fixed to be 0.1 . The critical value of $\dot{M}$ at a given radius, at which the disk returns in quiescence, depends on the radius in the accretion disk (equation (39) in Hameury et al. 1998), and the mass accretion rate in outburst would become large in long orbital period systems. We set values of $\dot{M}$ as $2 \times 10^{-9}, 3 \times 10^{-9}$, 
$4 \times 10^{-9}, 5 \times 10^{-9}, 6 \times 10^{-9} M_{\odot} \mathrm{yr}^{-1}$ for 1 SWASP J1621 and $\mathrm{BD} \mathrm{Pav}^{6}$ and $6 \times 10^{-8}, 8 \times 10^{-8}, 1 \times 10^{-7} M_{\odot} \mathrm{yr}^{-1}$ for $\mathrm{V} 364$ Lib, considering their orbital period. Also, we assumed the flat temperature distribution of a disk in quiescence $(3,000$ or 3,500 or 4,000 or 4,500K), referring to Wood et al. (1986).

On the basis of the assumption of the steady state, we set the thickness of accretion disk in outburst as follows:

$\frac{H}{r}=1.72 \times 10^{-2} \alpha^{-1 / 10} \dot{M}_{16}^{3 / 20}{\frac{M}{M_{\odot}}}^{3 / 8} r_{10}^{1 / 8}\left[1-\left(\frac{r_{\text {in }}}{r}\right)^{1 / 2}\right]^{3 / 5}(2)$

where $H, \dot{M}_{16}$ and $r_{10}$ represent the height of the disk, $\dot{M} / 10^{16}$ $\mathrm{g} \mathrm{s}^{-1}$ and $r / 10^{10} \mathrm{~cm}$, respectively (Shakura, Sunyaev 1973). On the other hand, we assumed that the disk is flat in quiescence for simplicity. The tested parameters of the height of the disk were $0.003,0.005,0.007,0.009$ in units of the binary separation.

The masses of the primary and the companion are fixed. We used the values in Table 1 . The radii of the white dwarfs in 1SWASP J1621, BD Pav, and V364 Lib are estimated to be $0.009,0.008$, and $0.008 R_{\odot}$, respectively (Nauenberg 1972). We assumed that the temperature of the companion star is 4,300 $\mathrm{K}$ for 1SWASP J1621, 3,500 K for BD Pav, and 6,600 K for V364 Lib, considering the spectral types of the companions (Allen 1973) and the reasoning in Sec. 4. The temperature of the white dwarfs is fixed to $20,000 \mathrm{~K}$. In addition, we need to set the distance from Earth to the object in order to determine the apparent magnitude.

\subsubsection{Limitation of Our Model and How to Determine the Best Model Parameters}

Our modeling deals with only the emission from optically-thick regions and assumes a simple geometry, an axisymmetric disk, no hot spot and no hot corona. The hot spot is located near the disk edge, and an optically-thin hot corona expanding around the disk is produced by the evaporation of the inner disk at low accretion rate. These components, which are missing from our models, have high temperature ranging between $\sim 5,000$ 20,000 K (Liu et al. 1995; Stanishev et al. 2001). In quiescence and/or in high inclination systems, therefore, these opticallythin hot regions are likely dominant, and hence, our models have some difficulties reproducing the observations, especially in the $B$ band. In other words, the observations in outburst in the $R_{\mathrm{C}}$ band are expected to be reproduced more easily in our models than those at shorter wavelengths. In addition, we assume that the distribution of the disk temperature in outburst follows that in the steady state, but the real distribution may not. The lack of a hot spot, simple disk geometry, and simple distribution of the disk temperature in the models are also raised in Zola et al. (2017) as the factors responsible for not completely

\footnotetext{
${ }^{6}$ If $\dot{M}$ is less than $1 \times 10^{-9} M_{\odot} \mathrm{yr}^{-1}$, the outer disk cannot be in the outburst state (equation (39) in Hameury et al. 1998).
}

explaining the observations.

On the basis of the limitations described in the previous paragraph, we gave priority to reproducing the $R_{\mathrm{C}}$-band phaseaveraged profile when determining the best models of 1SWASP J1621. In addition, we note that the inclination must have the same value in outburst and quiescence. We thus chose the best models as for 1SWASP J1621 in the following way.

1. We estimated at first each $\chi^{2}$ value between the observational and calculated orbital phases in the outburst state with various tested parameters specified in Sec. 5.2.2, and determined the best model in that state by the minimum $\chi^{2}$ estimations in the $R_{\mathrm{C}}$ band.

2. With the inclination fixed to its value in the best model in outburst, we estimated each $\chi^{2}$ value between the observational and calculated orbital phases in quiescence. We determined the best model by the minimum $\chi^{2}$ estimations in the $R_{\mathrm{C}}$ band.

As for BD Pav and V364 Lib, we chose the best model in quiescence by the minimum $\chi^{2}$ values between the observed $V$-band phase-averaged profile and the calculated $V$-band one. In addition, we chose the best mass accretion rate, which can best reproduce the outburst amplitude in the $V$ band, with the disk radius and inclination fixed as the best-fit parameters in quiescence.

It is difficult to employ sophisticated methods to calculate error bars due to the long computational times; therefore, we estimated the model profiles with the rough grids described in Sec. 5.2.2, and determined the $90 \%$ confidence intervals as the range in which $\Delta \chi^{2}$ from the minimum $\chi^{2}$ is within 2.706 , by computing the models in the finer grids than those described in Sec. 5.2.2. Since accurate constraints of the inclination and disk height in quiescence is beyond our current goals for 1SWASP J1621 and BD Pav, we fixed the inclination to the value which produces the minimum $\chi^{2}$ in these two objects. Additionally, it is difficult to restrict the ranges of the parameters which are related to the accretion disk in V364 Lib because of the bright companion; hence, we only provided an error bar for the inclination.

\subsection{Results}

Our modeling suggests the inclination is close to $90 \mathrm{deg}$ in 1SWASP J1621 (see Table 2). The best-fit models are displayed in figure 14 . The very high inclination has been already noticed by Kjurkchieva et al. (2017) and Zola et al. (2017). Our models do not accurately fit the phase profiles in quiescence and in the $V$ and $B$ bands in outburst, but they do reproduce the main characteristics of quiescent double-waved modulations without a hot companion. The models in Kjurkchieva et al. (2017) and Zola et al. (2017) for 1SWASP J1621 also did not reproduce the observations in all of the bands. Although our model includes 
Table 1. Properties of 1SWASP J1621, BD Pav, and V364 Lib.

\begin{tabular}{llcccc}
\hline Object & $P_{\text {orb }^{*}}$ & $M_{1}{ }^{\dagger}$ & $q^{\ddagger}$ & Outburst History $^{\S}$ & Reference $^{\|}$ \\
\hline 1SWASP J1621 & $0.207852(1)$ & 0.9 & 0.44 & 2006,2016 & $1,2,3$ \\
BD Pav & 0.17930 & 1.0 & 0.44 & $1938,1985,1996,1997,1998,2000,2006,2015,2017$ & $4-10$, This work \\
V364 Lib & $0.70243(11)$ & $1.0 \pm 0.2$ & $1.5 \pm 0.3$ & $2003,2006,2009,2012,2017$ & $11-16$, This work \\
\hline
\end{tabular}

${ }^{*}$ Orbital period in units of $\mathrm{d}$.

${ }^{\dagger}$ Mass of the primary white dwarf in units of $M_{\odot}$.

${ }^{\ddagger}$ Mass ratio of the companion star to the primary $\operatorname{star}\left(q \equiv M_{2} / M_{1}\right)$.

$\S$ Years when outbursts were recorded.

"1: Thorstensen (2016), 2: Drake et al. (2014a), 3: Kjurkchieva et al. (2017), 4: Sion et al. (2008), 5: Friend et al. (1990), 6: Barwig, Schoembs (1987), 7: vsnet-alert 1008, 8: vsnet-alert 2388, 9: vsnet-alert 4888, 10: vsnet-alert 18762, 11: Uemura et al. (2009), 12: vsnet-alert 14271, 13: Wils (2011), 14: Pojmanski et al. (2009), 15: Kinugasa et al. (2009), 16: vsnet-alert 20877.

more reasonable thicknesses of disks and temperature distributions of quiescent disks, compared to their models, it still had difficulty reproducing the quiescent profiles. This would be due to the limitations of our modeling, which are described in the first paragraph in Sec. 5.2.3. In particular, our model does not include any optically-thin region which is observable in highinclination systems and in quiescence. In addition, a hot spot would be dominant around the phase 0.75 in the $B$ band. Also, the calculated depth of the eclipse in the $B$ band in outburst is $\sim 0.5$-mag deeper than the observed one. Moreover, the calculated $B-V$ color is larger by $\sim 0.1 \mathrm{mag}$, and the calculated $V-R$ color was smaller by $\sim 0.15$ mag than the observed one. Some fine-tuning of the disk temperature and structure may help to reproduce completely the observational depth of the eclipse and colors, but this is beyond our purpose. Since this system seems to have a very high inclination, there is a possibility that a flared disk structure may contribute to the emission. If the inclination is close to $90 \mathrm{deg}$, the hot white dwarf and the inner disk are hidden by the outer disk, as shown in the upper panel of figure 17. In this case, the radiation from the hot white dwarf and the innermost region of the disk hardly contributes the overall emission from the object. This situation will easily produce the shallow primary minima in quiescence.

As for BD Pav, the inclination seems to be close to 75 deg (see Table 2), which is consistent with previous studies (Sion et al. 2008; Friend et al. 1990) and the estimation in Sec. 5.1. Since our modeling does not explain the observations well enough to estimate error bars, we just provide the best model parameter in the rough grids. The reasons why our model does not completely reproduce the observations are the limitations described in Sec. 5.2.3; in addition, our sparse profile may make it difficult to recognize the eclipse of the white dwarf in this object. The best-fit model in quiescence which is given in figure 15, however, reproduces the W UMa-like orbital modulations without a hot companion as in 1SWASP J1621. The mass accretion rate to reproduce the outburst amplitude is in the range expected by the disk-instability model. In addition, according to Barwig, Schoembs (1987), the $B-V$ colors in the outburst and quiescent states are $\sim 0.1$ and $\sim 0.6 \mathrm{mag}$, and the $V-R$ colors in the outburst and quiescent states are $\sim 0.0$ and $\sim 0.5$ mag, respectively; our modeling reproduces well the $V-R$ colors. Although the calculated $B-V$ color in quiescence was $\sim 0.6$ mag larger than the observational one, the reported colors in Barwig, Schoembs (1987) were measured at the end of the outburst. Some time after the outburst, the $B-V$ color might become large as the disk becomes cool. Though the grazing eclipses in the systems having $\sim 70$-deg inclinations are characterized by deep primary minima in quiescence as in U Gem (chapter 2.4.3 in Hellier 2001) due to bright hot spots, the eclipse in BD Pav does not agree with this behavior. This would be because of the dim hot spot as suggested in Sec. 5.1. If the inclination is $75 \mathrm{deg}$, the white dwarf is barely eclipsed (see also the middle panel of figure 17).

As for V364 Lib, we confirmed that the ellipsoidal variations of the hot companion are dominant in quiescence, and that the inclination is low (see Table 2). The estimated inclination is consistent with that in Sec. 4. The best-fit model is shown in figure 16. The accretion disk is not eclipsed, but the ellipsoidal variations are observable due to the hot companion star (see the lower panel of figure 17). As for the outburst state, the value of $\dot{M}$ is chosen to reproduce the outburst amplitude. The $\dot{M}$ value much larger than those in the other two objects seem to originate from its long orbital period. The critical value of $\dot{M}$ at which the transition from the hot branch to the cool branch on the thermal equilibrium curve is triggered, is approximately proportional to $r^{2.7}$ (Hameury et al. 1998) ${ }^{7}$. If the hot state extends to the outer disk in its outbursts, the mass accretion rate in outburst is likely much higher than those in shorter-period dwarf novae, in spite of our rough estimations. We also confirmed very weak ellipsoidal variations with amplitudes of less than 0.03 mag via the model with the parameters given in Table 2;

${ }^{7}$ Here, $r$ is the same one as defined in Sec. 5.2.2. 

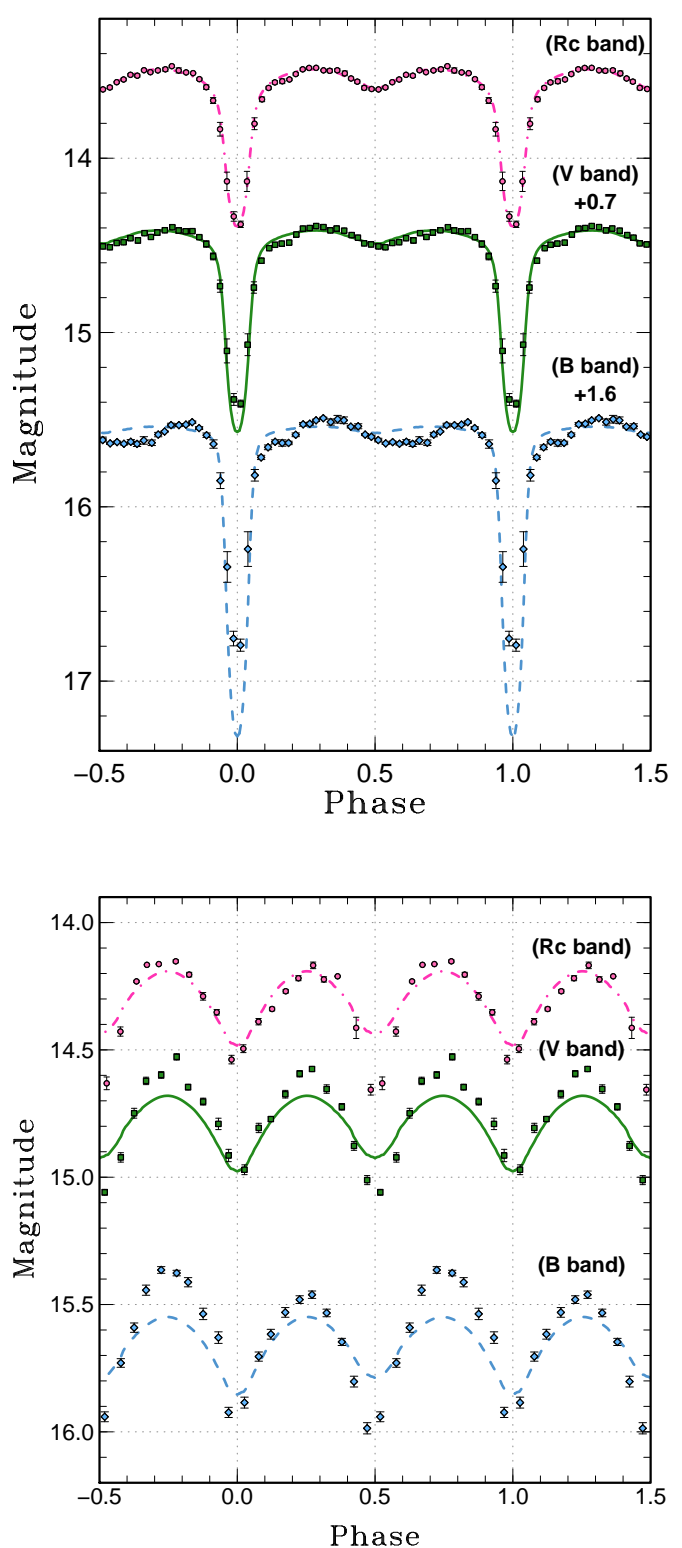

Fig. 14. Best models of the phase profiles of 1SWASP J1621 in outburst (upper panel) and quiescence (lower panel). The dot-dash, solid and dash lines represent the calculated phase profiles in the $R_{\mathrm{C}}, V$, and $B$ bands. The points with error bars are the observational phase profiles. The magnitudes of the observational phase profiles are offset for visibility except for those in the $R_{\mathrm{C}}$-band ones in outburst.

this is consistent with our observations. The reason for the lack of prominent ellipsoidal variations in outburst is that the disk becomes much brighter in the outburst than that in quiescence. We consider that the bright disk in outburst reduces the relative contribution of the ellipsoidal variations of its companion star, and that some variations hidden by the bright companion during quiescence become visible in outburst. In addition, the outburst amplitude is reproduced with the parameter in Table 2. The colors of $V-R$ and $B-V$ in quiescence are reported to be 0.1 and $0.5 \mathrm{mag}$, respectively, by Henden et al. (2016),

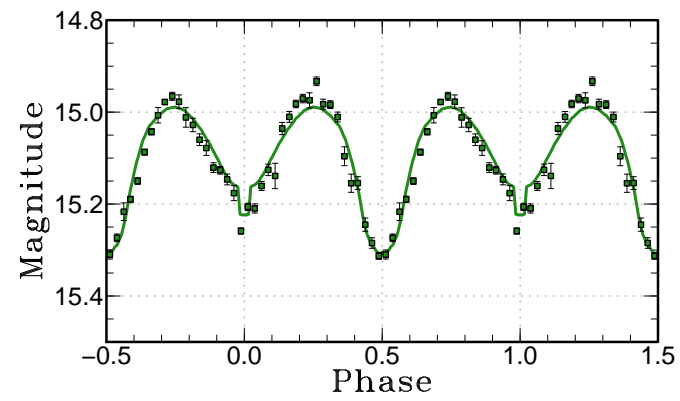

Fig. 15. Best model of the phase profile of BD Pav in quiescence. The solid line represents the calculated phase profile in the $V$ band. The points with error bars are the observational phase profile.

and our calculated colors were almost identical. In the systems having a high-temperature companion, its contribution dominates the whole emission from the dwarf nova. If these systems have high inclinations, the eclipses of companions may become deeper than those of the disks and white dwarfs in quiescence, and the eclipses of disks and white dwarfs are prominent in outburst. These are inconsistent with the observations of V364 Lib.

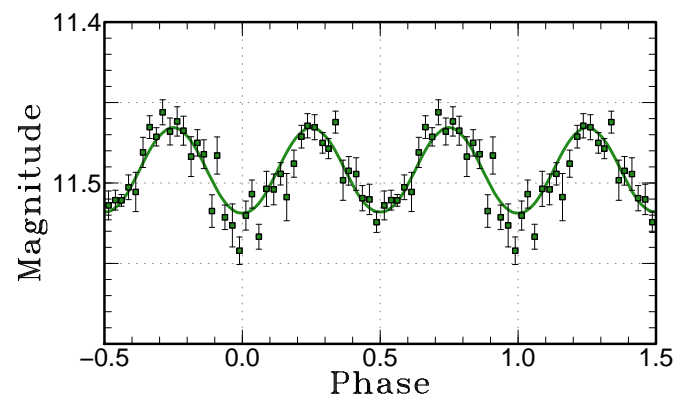

Fig. 16. Best model of the phase profile of $\mathrm{V} 364 \mathrm{Lib}$ in quiescence. The solid line represents the calculated phase profile in the $V$ band. The points with error bars are the observational phase profile.

\section{Discussion}

\subsection{Nature of the Three Objects and Their Low-Amplitude Outbursts}

Although 1SWASP J1621, BD Pav, and V364 Lib show similar outburst behavior, their natures are not necessary the same. As mentioned in the introduction, some people consider 1SWASP J1621 to have an unusually hotter companion star than normal $\mathrm{K}$ or M-type companions in dwarf novae (see Figure 2.45 in Warner 1995) according to Waagen (2017), but our results show that the characteristics of the orbital phase profiles of 1SWASP J1621 can be explained by its very high inclination and faint hot spot, without a hot companion star. This condition is similar to that in BD Pav. On the other hand, for V364 Lib, our results support that this system has a low inclination and an F-type hot companion star, which is expected to be bright (large) by the 

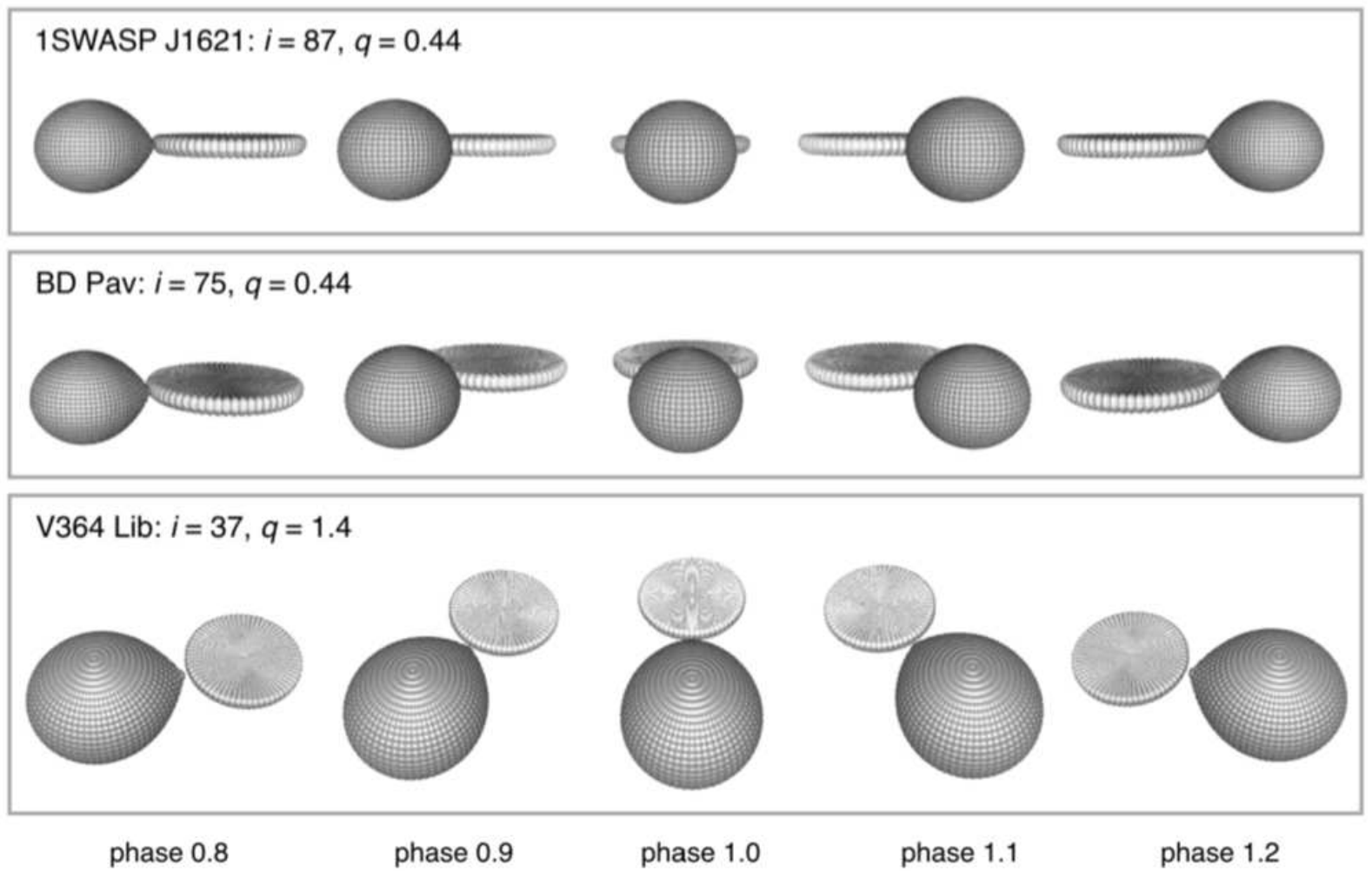

Fig. 17. Model configurations around the eclipses of the accretion disks in 1SWASP J1621, BD Pav, and V364 Lib in the outburst state.

long orbital period and the large mass ratio (see also Sec. 4).

Their properties are likely relevant to their small-amplitude ( 1-2 mag) outbursts (see Sec. 3). In the cases of 1SWASP J1621 and BD Pav, the critical conditions are their high inclinations and inside-out outbursts. In high-inclination systems, the observable disk surface is small. In addition, if a system has a very high inclination, as in 1SWASP J1621, the hot inner region of the accretion disk is hidden by the outer disk located along the line of sight. Although the inner disk becomes bright in the outburst state, it cannot be observed. The inside-out outbursts expected from the slow-rise shapes of the outbursts (see Sec. 3) would be another key factor of the low-amplitude outbursts. The outburst amplitudes of inside-out outbursts are often smaller than those of outside-in outbursts, since the slowly propagating heating front prevents the whole disk from entering the hot state (Smak 1984; Cannizzo et al. 1986). On the other hand, in V364 Lib, its hot companion star is the main factor in its low-amplitude outbursts, though inside-out outbursts may also contribute to the small outburst amplitudes. If the companion is unusually hot and big, its contribution to the whole brightness is large at optical wavelengths. The change of the disk brightness between the quiescence and outburst states would be unremarkable at optical wavelengths even if the total luminosity emitted from the disk is high in outburst. We suggest that astronomical surveys might easily overlook such a low-amplitude outburst.

\subsection{Mass-Transfer Bursts?}

We believe that the outbursts in the three objects are explained well by the disk-instability model. Qian et al. (2017) claimed the mass transfer ceases because of the star spot on the surface of the companion star, which is located at the $L_{1}$ point in quiescence, and the outbursts are caused by an abrupt mass transfer because of the expanding companion in 1SWASP J1621. However, our results show the existence of the large accretion disk in the outburst state in this object, and this is inconsistent with their argument. If a lot of matter is transferred to the primary side at the onset of the outburst, the radius of the accretion disk becomes close to the Lubow-Shu radius (Lubow, Shu 1975), which is approximately expressed by $0.0859 q^{-0.426}$ in units of the binary separation (Hessman, Hopp 1990), because of the conservation of angular momentum (Ichikawa, Osaki 1992). This value is much smaller than the estimated disk size in our modeling. When a small disk is occulted by the companion, the shape of the bottom of primary minima becomes flat, and this phenomenon disagrees with the morphology of the phase profiles observed in the outburst state. In addition, continuous accretion during quiescence may help to build a large disk in outburst by removing matter with small specific angular momentum. Zola et al. (2017) also concluded that their modeling of the eclipsing profile in quiescence suggests a white dwarf plus a large accretion disk, and it is consistent with our results 
Table 2. Model parameters to reproduce the eclipsing light variations and computed outburst amplitudes and colors in 1SWASP J1621, BD Pav, and V364 Lib.

\begin{tabular}{cccc}
\hline Parameters & 1SWASP J1621 & BD Pav & V364 Lib \\
\hline$i^{*}$ & 87 & 75 & $37_{-4}^{+1}$ \\
$R_{\text {disk }}^{\text {burst }} \dot{M}^{\ddagger}$ & $0.89_{-0.04}^{+0.01}$ & 0.90 (fix) & 0.80 (fix) \\
$R_{\text {disk }}^{\text {qui }}$ & $5.4_{-0.3}^{+0.1} \times 10^{-9}$ & $2 \times 10^{-9}$ & $1 \times 10^{-7}$ \\
$T_{\text {disk }} \|$ & $0.86_{-0.01}^{+0.04}$ & 0.90 & 0.80 \\
$h_{\text {disk }}{ }^{\#}$ & $4,470_{-70}^{+30}$ & 3,000 & 4,500 \\
$d^{\uparrow}$ & 0.009 & 0.007 & 0.003 \\
\hline Amp $^{* *}$ & 166 & 253 & 536 \\
$V-R_{\text {out }}{ }^{\dagger \dagger}$ & 0.70 & 3.11 & 0.85 \\
$B-V_{\text {out }}{ }^{\ddagger \ddagger}$ & 0.23 & 0.10 & 0.06 \\
$V-R_{\text {qui }}{ }^{\S \S}$ & 0.49 & 0.04 & 0.05 \\
$B-V_{\text {qui }}{ }^{*}$ & 0.87 & 0.73 & 0.20 \\
\hline
\end{tabular}

${ }^{*}$ Inclination angle in units of deg.

${ }^{\dagger}$ Disk size in the outburst state in units of $R_{\mathrm{L} 1}$.

${ }^{\ddagger}$ Mass accretion rate in outburst in units of $M_{\odot} \mathrm{yr}^{-1}$. We assume the steady state in outburst.

${ }^{\S}$ Disk size in quiescence in units of $R_{\mathrm{L} 1}$.

"Temperature of a disk in quiescence in Kelvin.

\# Thickness of a disk in units of its binary separation.

"Distance from Earth to the object, which reproduces the apparent magnitude in the $R_{\mathrm{C}}$ band in outburst as for 1SWASP $\mathrm{J} 1621$, and in the $V$ band in quiescence as for BD Pav and V364 Lib, in units of pc.

** Outburst amplitude in the $R_{\mathrm{C}}$ band at phase 0.25 in units of magnitude. It is derived from the best models in outburst and quiescence.

${ }^{\dagger \dagger} V-R$ color in outburst at phase 0.25 in units of magnitude. $\sharp \ddagger B-V$ color in outburst at phase 0.25 in units of magnitude. ${ }^{\S} V-R$ color in quiescence at phase 0.25 in units of magnitude.

${ }^{\|I\|} B-V$ color in quiescence at phase 0.25 in units of magnitude.

in Sec. 5.3. Their results, and ours, argue against the halting of mass transfer in quiescence, which is a key element in Qian et al. (2017). Therefore, there is no need for introducing mass transfer bursts to explain the outbursts in this kind of object. Qian et al. (2017) proposed their interpretation based in part on the observations of Pavlenko et al. (2016), but the short-term periodic variations reported in this reference were not confirmed in our observations.

Actually, there are some contradictions between the observations and the discussion in Qian et al. (2017). For example, they considered that only the eclipse of the white dwarf was observed in quiescence, but the width of the eclipse that they regard as the ingress of the white dwarf, about $0.007 \mathrm{~d}$, indicates that the eclipsed object must have a width of more than 10 times of the radius of a $0.9-M_{\odot}$ white dwarf. $^{8}$ In addition, the depth of the eclipse, about $0.2 \mathrm{mag}$, is not consistent with the eclipse of a white dwarf. If the temperature of the white dwarf is $20,000 \mathrm{~K}$, the contribution of the white dwarf to the brightness of the entire system is about $10 \%$. The depth would then be less than 0.1 mag in their observations. Since they consider that accretion onto the white dwarf is stopped during quiescence, the white dwarf should have a low-temperature in their discussion. If the temperature of the white dwarf is only $10,000 \mathrm{~K}$, the depth of eclipses would be less than $0.01 \mathrm{mag}$. Moreover, they insisted no lasting disk in this object. If the contribution of the disk is very weak and the disk size is small in outburst, the flux at the primary minima in outburst would be the same as those in quiescence; however, this is inconsistent with figure 3 in Qian et al. (2017).

\subsection{Infrequent, Long-lasting and Inside-out Outbursts}

Though we may overlook the outbursts due to their small amplitudes, the frequency of the outbursts in these systems is relatively low in comparison with many other dwarf novae above the period gap (see also Table 1), and their outburst durations are relatively long. We discuss below the origin of the infrequent, long-lasting, and inside-out outbursts.

As for the origin of the infrequent outbursts in 1SWASP J1621 and BD Pav, we consider that it is attributed to low masstransfer rates. This is because 1SWASP J1621 and BD Pav have clearly longer outburst intervals than many other dwarf novae having 4-5 hours orbital periods. In addition, the almost symmetrical profiles with respect to the primary minima in these two objects (see figures 3 and 5) suggest that the hot spots are faint. This implies that the mass transfer rate is low, since the brightness of a hot spot depends on it. On the other hand, the origin of the infrequent outbursts in V364 Lib would not be necessarily low mass transfer rate. We suspect its long orbital period is related to the origin. This is because it would take much time to form a dense disk enough to trigger outbursts in V364 Lib due to its long orbital period which means a large value of $R_{\mathrm{L} 1}$. The relation between long outburst intervals and long orbital periods in several dwarf novae has been pointed out in Bruch (2017), and we show it in figure 18. ${ }^{9}$ For instance, compared with an typical dwarf nova having an orbital period of 4 hours, a mass ratio of 0.45 and outburst intervals of 30-100

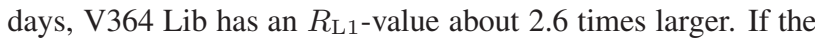

${ }^{8}$ The width of the ingress of the white dwarf is derived from the data in figure 2 in Qian et al. (2017).

${ }^{9}$ The values of outburst intervals in the long-period objects were derived from Menzies et al. (1986); Shears, Poyner (2009); Sekiguchi (1992); Bruch (2017) and see also figure E1 in the supplementary information of this paper. V1017 Sgr also has a very long orbital period, but we excluded it from the regression shown in figure 18 because it is clearly out of the trend. 
transfer rate is almost equal to that in a typical dwarf nova, then the accretion disk in V364 Lib would accumulate 2.5-7-times more mass than that in a typical dwarf nova until an outburst is triggered. In other words, based on mass transfer in ordinary dwarf novae, we would expect V364 Lib to have an outburst interval of about 70-700 days, but this value seems to be shorter than the observational outburst intervals in V364 Lib. Actually, V364 Lib may not follow the linear relation between the orbital period and the logarithm of the outburst intervals in these systems, although all outbursts in this object might not be detected due to their small amplitudes. Since the above discussion is a simple approach, our estimation has some uncertainty. ${ }^{10}$ We thus could not reject either of the long orbital period or the low mass-transfer rate as the origin of the infrequent outbursts in V364 Lib. The value of $\dot{M}$ in Table 2 represents not the masstransfer rate but the accretion rate in outburst. The actual mass transfer rate should be smaller than that value.

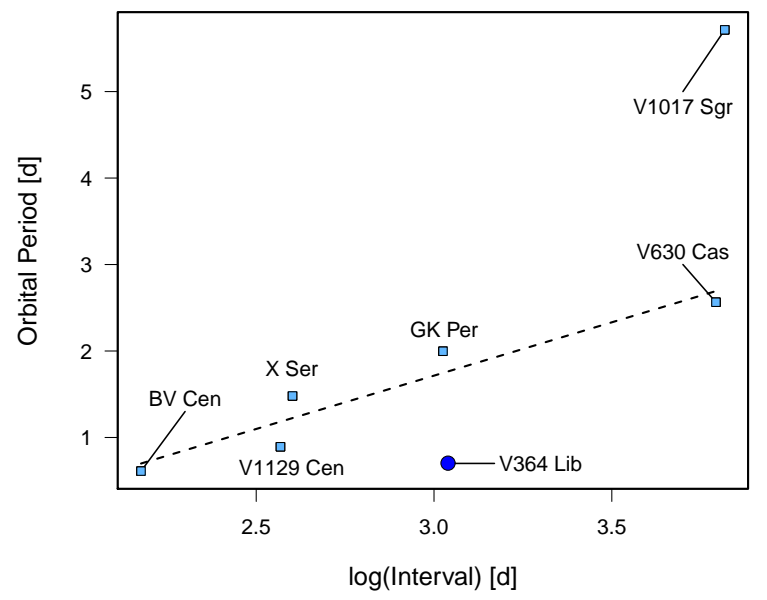

Fig. 18. Linear relation between the orbital periods and the logarithm of the outburst intervals in several dwarf novae having very long orbital periods. The dashed line represents the regression formula $y=1.2 x-2.0$ estimated by the least-squares method with the data of BV Cen, V1129 Cen, GK Per, $X$ Ser and V630 Cas. Here, $x$ and $y$ represent the orbital period and the interval between outbursts.

The inherent low-mass transfer rates and/or long orbital periods would naturally explain the characteristic long outburst durations and inside-out outbursts in the three objects within the context of the disk instability model. This is because a lot of mass is expected to accumulate in the disk and because the

\footnotetext{
${ }^{10}$ For example, the mass transfer rate is likely larger in the systems having longer orbital periods so that the rate in this object might be about 4 times larger than that in an typical dwarf nova according to equations (38) and (39) in Hameury et al. (1998). It is, however, possibly similar to the typical one, because this system enters inside-out outbursts. In addition, the structure and density profile in the disk would also affect the outburst intervals, and the expected intervals may become longer.
}

mass would be easily diffused inward on viscous timescales in these conditions. Actually, the numerical simulations of the disk instability model showed that, in the dwarf nova GK Per having a very long orbital period, most of its outbursts are easily triggered at the inner disk, and that this behavior cannot be reproduced by mass-transfer bursts (Kim et al. 1992). It is also consistent to the idea of the disk-instability model that the large stored disk mass before the onset of an outburst can be related to the long outburst durations. The slow rises of these outbursts, in addition to their small amplitudes, may also make it difficult to detect them. Long-lasting inside-out outbursts were commonly observed in V1129 Cen and HS 0218, which are similar to V364 Lib and 1SWASP J1621, respectively (Bruch 2017; Golysheva et al. 2012); Golysheva et al. (2012) have already pointed out that an inside-out outburst is expected from systems with low mass-transfer rates, which is considered to be associated with the almost symmetrical phase profiles, as in HS 0218.

The mechanism that causes lower mass-transfer rates in the three objects than those in many other dwarf novae above the period gap may be unclear. One plausible explanation is that the mass-transfer rate varies in the same system on much longer timescales than the interval between outbursts for some reason. For example, Shara et al. (1986) and Kovetz et al. (1988) proposed hibernation scenario. According to their proposition, during about a century after a nova eruption, the mass-transfer rate remains high due to the strong irradiation from the white dwarf, and thereafter, it decreases. V1213 Cen, a classical nova, revealed dwarf-nova outbursts 6 years before its eruption (Mróz et al. 2016), and this phenomenon is understood as evidence of hibernation. The hibernation is, however, believed to occur only when the binary separation expands just after a nova eruption. On the basis of equations (14) and (21), which represent the relation between the variation of binary separation and the mass ratio in Shara et al. (1986), it is doubtful whether the binary separation expands after a nova eruption in V364 Lib. 1SWASP J1621, BD Pav may be in hibernation, but V364 Lib may not.

\subsection{Appearance of Highly lonized Emission Lines}

The He II 4686 emission line was observed in 1SWASP J1621, BD Pav, and V364 Lib during the outburst state (Scaringi et al. 2016; Barwig, Schoembs 1987; Sec. 4). In addition, the C III/N III emission line was also observed in the outburst of V364 Lib. These highly ionized lines are barely detected in the outbursts of many dwarf novae. In the case of V364 Lib, a massive white dwarf is a natural consequence on the basis of its massive companion star (see Sec. 4). Since the primary star has degenerated earlier, the progenitor of the primary white dwarf would be more massive than a F-type companion star as for V364 Lib. For example, the relation between the final whitedwarf mass and the initial main-sequence mass given in Fig. 2 
in Salaris et al. (2009) shows the white-dwarf mass can exceed $1 M_{\odot}$ if the initial mass exceeds $\sim 6 M_{\odot}$, and our observations do not deny the possibility that the initial mass of the primary exceeds $\sim 6 M_{\odot}$. It is thus suggested that the primary white dwarf in this system is likely heavier than those in the $\mathrm{CVs}$ which do not have massive donor stars. In spite of the large uncertainty, our estimation in Sec. 4 implies the possibility that this object has a massive (more than $1 M_{\odot}$ ) white dwarf. If the white dwarf is massive, the inner disk has a relatively high temperature. The massive white dwarf may be, therefore, the origin of the highly ionized emission lines in this system. We note that some dwarf novae having massive white dwarfs showed highly-ionized emission lines in outburst (e.g., Wilber et al. (2015); Wargau (1988); Morales-Rueda, Marsh (2002)). In addition, this type of line was detected in a recurrent nova having a massive white dwarf (Diaz et al. 2010).

On the other hand, the source to produce the He II emission line in outburst in normal dwarf novae having $\sim 0.8-M_{\odot}$ white dwarfs (Zorotovic et al. 2011) is unclear, but such a highly ionized emission line is sometimes observed in high inclination systems (e.g., Baba et al. 2002; Harlaftis et al. 1992). The He II emission line in 1SWASP J1621 and BD Pav may arise due to their high inclinations, although their relatively massive white dwarfs, heavier than $\sim 0.8-M_{\odot}$, may contribute to the line as in V364 Lib. This possibility is also notified by Zola et al. (2017). In the high-inclination systems, the accretion disk is optically thick. Additionally, the He II emission line in the 1985 outburst of BD Pav showed a single-peaked profile according to figure 1 in Barwig, Schoembs (1987), although our modeling and other works suggest the inclination of this system is $\sim 75 \mathrm{deg}$. The emission line from the accretion disk will have a clear doublepeaked shape due to its high inclination (Horne, Marsh 1986). Thus the radiation is not likely to originate from a disk. Such a single-peaked He II emission line was also observed in outburst in HT Cas, another dwarf nova, and a disk wind is proposed as its possible origin (Murray, Chiang 1997). In the outbursts of dwarf novae, the disk temperature becomes high as in novalike systems. The single-peaked emission lines whose origin is interpreted as the disk wind are often observed in nova-like systems (e.g., Baptista et al. 2002), and hence, it would not be strange that the strong single-peaked emission lines are detected in some dwarf-nova outbursts. Additionally, an optically-thin disk wind may contribute to the phase profile in the $B$ band.

\subsection{Evolutionary Stage of V364 Lib}

It seems to be strange at first glance that V364 Lib, whose mass ratio is likely more than 1 has low mass transfer rate. In a subclass of CVs which have a companion more massive than the primary white dwarf, if the companion star completely fills with its Roche lobe, the mass transfer becomes easily unstable since the mass loss from the companion star induces the binary separation to shrink. We call this subclass "super soft sources". In these systems, the mass transfer rate is as high as $1-4 \times 10^{-7}$ $M_{\odot} \mathrm{yr}^{-1}$ (see Kahabka, van den Heuvel 1997 for a review and references therein). This value is more than 100 times higher than the typical mass transfer rate in dwarf novae (Shafter et al. 1986). On the contrary, if we consider the whole amount of the stellar wind flows via the $L_{1}$ point under the condition that the radius of the companion star has not yet filled with its Roche lobe, the mass transfer rate is too low to trigger dwarf-novae type outbursts. Under the assumption that the wind velocity is the escape velocity, the mass transfer rate estimated to be $\sim 10^{-11-12} M_{\odot} /$ yr by using the equation $\dot{M}=4 \pi R_{2}{ }^{2} \rho v_{\text {wind }}$. We finally suggest that in V364 Lib, the thin outer layer of the companion fills with its Roche lobe. Since the estimated value of the Roche radius on the companion side is about twice of the radius of a typical main-sequence F-type star, the companion is likely a sub-giant if our suggestion is correct.

Although the mass transfer rate in V364 Lib seems to be low at present, it may increase as the companion evolves and/or the orbital angular momentum decreases. Then this system may show nova eruptions repeatedly since the white dwarf is likely massive (Fujimoto 1982). In addition, if the mass transfer becomes unstable (i.e., the transfer rate becomes close to $\sim 10^{-7} M_{\odot} / \mathrm{yr}$ ), the system behaves as a super soft source. V364 Lib might eventually become a supernova via the supersoft-source phase. The duration of the post-common-envelope phase, before the system evolves to a completely semi-detached binary, is likely long, since the companion star evolves on timescales of $\sim 10^{9} \mathrm{yr}$ and the orbital angular momentum is decreasing on timescales of $\sim 10^{8-9}$ yr (e.g., figure 12 in Knigge et al. 2011). It would not be strange that some CV-like systems having massive companions show dwarf-nova-like outbursts. Recent numerical simulations on the $\mathrm{CV}$ evolution suggest that some CVs having F-type donor stars enter dwarf-nova type outbursts (figures 4 and 16 in Kalomeni et al. 2016).

\section{Conclusions}

We presented the optical photometric observations of 1SWASP J1621, BD Pav, and V364 Lib and the optical spectroscopy of V364 Lib, and investigated their properties by using the numerical modeling. Our main findings and discussion are summarized in the following descriptions.

- There are many common features in the outburst behavior and the orbital variability in 1SWASP J1621, BD Pav, and V364 Lib. They show infrequent and small-amplitude outbursts. The outburst durations are long (about a few tens of days), and slow rises in brightness are observed at an early stage. In addition, all of the three systems show prominent ellipsoidal variations in quiescence. 
- In spite of the similarities in the outburst behavior, some properties in each system are somewhat different from the others. Our observations and numerical modeling suggest that 1SWASP J1621 has a very high inclination ( 90 deg) plus a faint hot spot, and these properties are similar to those in BD Pav, though it has a little lower inclination ( $\sim 75 \mathrm{deg})$. On the other hand, V364 Lib likely has an F-type bright companion star, a massive white dwarf, and a low inclination angle.

- The characteristic low-amplitude outbursts in the three objects can be explained by a high inclination or a hot companion and inside-out outbursts.

- The almost symmetrical phase profiles with respect to the primary minima, which arise due to faint hot spots, and long intervals between outbursts, would suggest a low mass-transfer rate in 1SWASP J1621 and BD Pav. The long orbital period implies the large disk for V364 Lib. The low mass-transfer rate and/or the large disk may be related to long outburst intervals, long outburst durations, and inside-out outbursts in these three objects.

- The commonly observed, highly ionized emission lines in their outbursts would originate from the high inclinations, for 1SWASP J1621 and BD Pav, and from the massive white dwarf for V364 Lib. A disk wind may be related to the profile of these highly ionized emission lines.

- In spite of a donor star more massive than the primary white dwarf, V364 Lib shows dwarf-nova like outbursts. This implies only the thin outer layer of the donor star fills its Roche lobe in this system. This object may evolve into a recurrent nova or a super soft source.

Although the properties of the three objects are somewhat different from normal dwarf novae, (in particular, V364 Lib is different from many other dwarf novae having cool companions), our results show that the peculiar outburst behavior and the changing orbital profiles between outburst and quiescence in the three objects can be explained in terms of the disk instability model in normal dwarf novae. It is not necessary to consider strong magnetic activity and mass transfer bursts. The estimation of the mass ratio in V364 Lib has a large error, and hence, high-dispersion spectroscopy in the outburst state is important to determine it with high accuracy. We consider that the three systems recently reported by Bruch, Monard (2017); Bruch (2017); Golysheva et al. (2013) have properties similar to those of 1SWASP J1621, BD Pav, or V364 Lib, and our discussion is applicable to their peculiar outburst behavior. Although the total number of this kind of object may be large, it is difficult to find them due to their low-amplitude and rare outbursts.

\section{Acknowledgements}

This work was financially supported by the Grant-in-Aid for JSPS Fellows for young researchers (M. Kimura, R. Ishioka) and the Grant-in-Aid from the Ministry of Education, Culture, Sports, Science and Technology (MEXT) of Japan (25120007, T. Kato and 17917351, D. Nogami). We appreciate AllSky Automated Survey for Supernovae (ASAS-SN) detecting a large amount of DNe. We are thankful to many amateur observers for providing a lot of data used in this research. The numerical code that we used in this paper is provided by Izumi Hachisu. This work was partially supported by Grants VEGA 2/0008/17, APVV-15-0458 (S. Shugarov), NSh 9670.2016.2 (N. Katysheva, S. Shugarov), RSF-1412-00146 (P. Golysheva, for processing observations data). O. Vozyakova and S. Shugarov acknowledges (partial) support from M. V. Lomonosov Moscow State University Program of Development.

\section{Supporting information}

Additional supporting information can be found in the online version of this article: Supplementary tables E1, E2, E3, E4, E5, E6, and E7 and figure E1.

\section{References}

Allen, C. W. 1973, Astrophysical Quantities, third edition (Athlone Press: London)

Baba, H., et al. 2002, PASJ, 54, L7

Baptista, R., Haswell, C. A., \& Thomas, G. 2002, MNRAS, 334, 198

Barwig, H., \& Schoembs, R. 1983, A\&A, 124, 287

Barwig, H., \& Schoembs, R. 1987, The Messenger, 47, 19

Bruch, A. 2017, New Astron., 57, 51

Bruch, A., \& Monard, B. 2017, New Astron., 55, 17

Cannizzo, J. K., Wheeler, J. C., \& Polidan, R. S. 1986, ApJ, 301, 634

Castelli, F., \& Kurucz, R. L. 2004, arXiv:astro-ph/0405087

Cleveland, W. S. 1979, J. Amer. Statist. Assoc., 74, 829

Davis, A. B., Shappee, B. J., Archer Shappee, B., \& ASAS-SN 2015, American Astron. Soc. Meeting Abstracts, 225, \#344.02

Diaz, M. P., Williams, R. E., Luna, G. J., Moraes, M., \& Takeda, L. 2010, AJ, 140, 1860

Drake, A. J., et al. 2016, The Astronomer's Telegram, 9112

Drake, A. J., et al. 2014a, MNRAS, 441, 1186

Drake, A. J., et al. 2014b, ApJS, 213, 9

Fernie, J. D. 1989, PASP, 101, 225

Friend, M. T., Martin, J. S., Connon-Smith, R., \& Jones, D. H. P. 1990, MNRAS, 246, 637

Fujimoto, M. Y. 1982, ApJ, 257, 767

Golysheva, P., Katysheva, N., Shugarov, S., Borisov, N., \& Gabdeev, M. 2013, Central European Astrophys. Bull., 37, 345

Golysheva, P. Y., Antipin, S. V., Zharova, A. V., Katysheva, N. A., Chochol, D., \& Shugarov, S. Y. 2012, Astrophysics, 55, 208

Hachisu, I., \& Kato, M. 2001, ApJ, 558, 323

Hachisu, I., \& Kato, M. 2003a, ApJ, 588, 1003 
Hachisu, I., \& Kato, M. 2003b, ApJ, 590, 445

Hameury, J.-M., Menou, K., Dubus, G., Lasota, J.-P., \& Hure, J.-M. 1998, MNRAS, 298, 1048

Harlaftis, E. T., Naylor, T., Hassall, B. J. M., Charles, P. A., Sonneborn, G., \& Bailey, J. 1992, MNRAS, 259, 593

Hellier, C. 2001, Cataclysmic Variable Stars: How and why they vary (Berlin: Springer)

Henden, A. A., Templeton, M., Terrell, D., Smith, T. C., Levine, S., \& Welch, D. 2016, VizieR Online Data Catalog, 2336

Hessman, F. V., \& Hopp, U. 1990, A\&A, 228, 387

Horne, K. 1985, MNRAS, 213, 129

Horne, K., Gomer, R. H., \& Lanning, H. H. 1982, ApJ, 252, 681

Horne, K., \& Marsh, T. R. 1986, MNRAS, 218, 761

Ichikawa, S., \& Osaki, Y. 1992, PASJ, 44, 15

Kahabka, P., \& van den Heuvel, E. P. J. 1997, ARA\&A, 35, 69

Kalomeni, B., Nelson, L., Rappaport, S., Molnar, M., Quintin, J., \& Yakut, K. 2016, ApJ, 833, 83

Kato, T., et al. 2010, PASJ, 62, 1525

Katysheva, N., Shugarov, S., Borisov, N., Gabdeev, M., \& Golysheva, P. 2015, Acta Polytechnica CTU Proceedings, 2, 123

Katysheva, N., Shugarov, S., \& Golysheva, P. 2017, in Stars: From Collapse to Collapse, ed. Y. Y. Balega, D. O. Kudryavtsev, I. I. Romanyuk, \& I. A. Yakunin Vol. 510 of Astronomical Society of the Pacific Conference Series(. p. 413

Kim, S.-W., Wheeler, J. C., \& Mineshige, S. 1992, ApJ, 384, 269

Kinugasa, K., Honda, S., Hashimoto, O., Kato, T., Maehara, H., \& Nogami, D. 2009, Cent. Bur. Electron. Telegrams, 1774, 2

Kjurkchieva, D. P., Popov, V. A., Vasileva, D. L., \& Petrov, N. I. 2017, New Astron., 52, 8

Knigge, C., Baraffe, I., \& Patterson, J. 2011, ApJ, 194, 28

Kovetz, A., Prialnik, D., \& Shara, M. M. 1988, ApJ, 325, 828

Liu, F. K., Meyer, F., \& Meyer-Hofmeister, E. 1995, A\&A, 300, 823

Lohr, M. E., Norton, A. J., Kolb, U. C., Maxted, P. F. L., Todd, I., \& West, R. G. 2013a, A\&A, 549, A86

Lohr, M. E., Norton, A. J., Kolb, U. C., Maxted, P. F. L., Todd, I., \& West, R. G. 2013b, A\&A, 549, A86

Lubow, S. H., \& Shu, F. H. 1975, ApJ, 198, 383

Maehara, H. 2016, The Astronomer's Telegram, 9113

Menzies, J. W., O’Donoghue, D., \& Warner, B. 1986, Ap\&SS, 122, 73

Morales-Rueda, L., \& Marsh, T. R. 2002, MNRAS, 332, 814

Mróz, P., et al. 2016, Nature, 537, 649

Murray, N., \& Chiang, J. 1997, ApJ, 474, 91

Nauenberg, M. 1972, ApJ, 175, 417

Noguchi, K., et al. 2002, PASJ, 54, 855

Osaki, Y. 1996, PASP, 108, 39

Palaversa, L., et al. 2013, AJ, 146, 101

Pavlenko, E. P., Sosnovskij, A. A., \& Antonyuk, O. I. 2016, The Astronomer's Telegram, 9138

Pojmanski, G., \& Maciejewski, G. 2004, Acta Astron., 54, 153

Pojmanski, G., Szczygiel, D., \& Pilecki, B. 2009, Central Bureau Electronic Telegrams, 1774

Qian, S.-B., et al. 2017, ApJ, 848, 131

Salaris, M., Serenelli, A., Weiss, A., \& Miller Bertolami, M. 2009, ApJ, 692,1013

Scaringi, S., Mason, E., Van Winckel, H., \& Escorza, A. 2016, The Astronomer's Telegram, 9122

Sekiguchi, K. 1992, Nature, 358, 563

Shafter, A. W., Wheeler, J. C., \& Cannizzo, J. K. 1986, ApJ, 305, 261
Shakura, N. I., \& Sunyaev, R. A. 1973, A\&A, 24, 337

Shara, M. M., Livio, M., Moffat, A. F. J., \& Orio, M. 1986, ApJ, 311, 163

Shears, J., \& Poyner, G. 2009, Journal of the British Astronomical Association, 119, 165

Sion, E. M., Gänsicke, B. T., Long, K. S., Szkody, P., Knigge, C., Hubeny, I., deMartino, D., \& Godon, P. 2008, ApJ, 681, 543

Smak, J. 1984, Acta Astron., 34, 161

Stanishev, V., Kraicheva, Z., Boffin, H. M. J., \& Genkov, V. 2001, A\&A, 367,273

Stellingwerf, R. F. 1978, ApJ, 224, 953

Thorstensen, J. 2016, The Astronomer's Telegram, 9141

Uemura, M., et al. 2009, The Astronomer's Telegram, 2030

Waagen, E. O. 2017, AAVSO Alert Notice, 569

Wargau, W. F. 1988, Monthly Notes of the Astronomical Society of South Africa, 47, 31

Warner, B. 1987, MNRAS, 227, 23

Warner, B. 1995, Cataclysmic Variable Stars (Cambridge: Cambridge University Press)

Wilber, A., Neric, M., Starrfield, S., Wagner, R. M., \& Woodward, C. E. 2015, The Astronomer's Telegram, 7217

Wils, P. 2011, J. American Assoc. Variable Star Obs., 39, 60

Wood, J., Horne, K., Berriman, G., Wade, R., O’Donoghue, D., \& Warner, B. 1986, MNRAS, 219, 629

Zejda, M., \& Pejcha, O. 2016, The Astronomer's Telegram, 9132

Zola, S., et al. 2017, AJ, 154, 276

Zorotovic, M., Schreiber, M. R., \& Gänsicke, B. T. 2011, A\&A, 536, A42 
Table E1. List of Instruments.

\begin{tabular}{|c|c|c|c|}
\hline CODE $^{*}$ & Telescope (\& CCD) & Observatory (or Observer) & Site \\
\hline $\mathrm{BSM}$ & $25 \mathrm{cmSC}+$ Moravian G2-1600 & Flarestar Observatory & San Gwann, Malta \\
\hline $\mathrm{COO}$ & PF $74 \mathrm{~cm}$ reflector+SBIG STL1001E & Lewis Cook & California, USA \\
\hline GFB & CDK 50cm+Apogee U6 & William Goff & California, USA \\
\hline IMi & $35 \mathrm{cmSC}+\mathrm{SXVR}-\mathrm{H} 16$ & Ian Miller & Furzehill Observatory, UK \\
\hline Ioh & 30cmSC+ST-9XE CCD & Hiroshi Itoh & Tokyo, Japan \\
\hline Mhh & 10.5cm+SBIG ST-8XME & Hiroyuki Maehara & Japan \\
\hline Mic & $84 \mathrm{~cm}+\mathrm{E} 2 \mathrm{~V}$ 42-40 & San Pedro Martir Observatory & Baja California, Mexico \\
\hline \multirow[t]{2}{*}{ MLF } & $30 \mathrm{cmRCX} 400+\mathrm{ST} 8-\mathrm{XME}$ & Berto Monard Calitzdorp & South Africa \\
\hline & $35 \mathrm{cmRCX} 400+\mathrm{ST} 8-\mathrm{XME}$ & Berto Monard Calitzdorp & South Africa \\
\hline $\mathrm{OKU}$ & $51 \mathrm{~cm}+$ Andor DW936N-BV & OKU Astronomical Observatory & Osaka, Japan \\
\hline SGE & 43cmCDK+STXL-11002 & Sierra Remote Observatories & Auberry, CA, USA \\
\hline \multirow[t]{4}{*}{ SHU } & 18/225cm Maksutov telescope+SBIG ST-10XME & Stara Lesna & Slovakia \\
\hline & 60/750cm Zeiss telescope+FLI ML 3041 & Stara Lesna & Slovakia \\
\hline & 50/200cm Maksutov telescope+Apogee Alta U16M & $\begin{array}{l}\text { Crimean Stations of Sternberg } \\
\text { Astronomical Institute (SAI) }\end{array}$ & Crimea \\
\hline & $250 / 2000 \mathrm{~cm}+\mathrm{LNI}$ BSI NBI $4 \mathrm{k} * 4 \mathrm{k}$ & $\begin{array}{c}\text { Caucasian Mountain Observatory } \\
\text { KGO (SAI, KGO) }\end{array}$ & The North Caucasus, Russia \\
\hline SRI & CDK 43cm+SBIG STL-1001 & Richard Sabo & Montana, USA \\
\hline Trt & 25cm ALCCD5.2 (QHY6) & Tamás Tordai & Budapest, Hungary \\
\hline
\end{tabular}


Table E2. Log of observations of the 2016 outburst of 1 SWASP J1621.

\begin{tabular}{|c|c|c|c|c|c|c|}
\hline Start* & End* & $\operatorname{Mag}^{\dagger}$ & Error $^{\ddagger}$ & $N^{\S}$ & Obs ${ }^{\|}$ & Band $^{\#}$ \\
\hline 466.9381 & 467.0381 & 14.705 & 0.015 & 47 & Mic & $R_{\mathrm{C}}$ \\
\hline 466.9387 & 467.0365 & 15.341 & 0.017 & 46 & Mic & $V$ \\
\hline 466.9395 & 467.0373 & 16.254 & 0.026 & 46 & Mic & $B$ \\
\hline 468.9367 & 468.9931 & 14.845 & 0.027 & 26 & Mic & $R_{\mathrm{C}}$ \\
\hline 468.9373 & 468.9937 & 15.472 & 0.029 & 26 & Mic & $V$ \\
\hline 468.9382 & 468.9922 & 16.348 & 0.029 & 25 & Mic & $B$ \\
\hline 505.7052 & 506.0115 & 16.268 & 0.016 & 137 & Mic & $B$ \\
\hline 505.7060 & 506.0101 & 14.750 & 0.013 & 136 & Mic & $R_{\mathrm{C}}$ \\
\hline 505.7066 & 506.0107 & 15.379 & 0.014 & 136 & Mic & $V$ \\
\hline 542.8993 & 542.9831 & 13.053 & 0.006 & 38 & Mic & $R_{\mathrm{C}}$ \\
\hline 542.8999 & 542.9837 & 13.382 & 0.007 & 38 & Mic & $V$ \\
\hline 542.9007 & 542.9846 & 13.818 & 0.009 & 38 & Mic & $B$ \\
\hline 543.6787 & 543.9624 & 13.501 & 0.014 & 812 & SGE & $C V$ \\
\hline 543.8746 & 543.9054 & 14.243 & 0.048 & 34 & HBB & $V$ \\
\hline 544.6798 & 544.9507 & 13.804 & 0.020 & 400 & SGE & $C V$ \\
\hline 544.7206 & 544.8464 & 13.786 & 0.016 & 313 & $\mathrm{COO}$ & $C V$ \\
\hline 544.8811 & 544.9792 & 13.519 & 0.052 & 44 & Mic & $R_{\mathrm{C}}$ \\
\hline 544.8817 & 544.9776 & 13.925 & 0.062 & 43 & Mic & $V$ \\
\hline 544.8826 & 544.9784 & 14.449 & 0.079 & 43 & Mic & $B$ \\
\hline 545.3104 & 545.5287 & 1.759 & 0.013 & 485 & CRI & $C V$ \\
\hline 545.4019 & 545.5645 & 13.891 & 0.026 & 219 & $\mathrm{RPc}$ & $V$ \\
\hline 545.4241 & 545.5385 & 13.875 & 0.007 & 66 & SHU & $C V$ \\
\hline 545.6793 & 545.9420 & 13.885 & 0.016 & 300 & SRI & $C V$ \\
\hline 545.7179 & 545.9854 & 14.102 & 0.022 & 362 & SGE & $C V$ \\
\hline 545.7365 & 545.9182 & 13.489 & 0.010 & 877 & $\mathrm{COO}$ & $R_{\mathrm{C}}$ \\
\hline 546.3322 & 546.4039 & 14.259 & 0.041 & 101 & Trt & $V$ \\
\hline 546.4068 & 546.6473 & 13.621 & 0.023 & 150 & Mic & $R_{\mathrm{C}}$ \\
\hline 546.4071 & 546.6477 & 14.056 & 0.027 & 150 & Mic & $V$ \\
\hline 546.4078 & 546.6483 & 14.624 & 0.038 & 150 & Mic & $B$ \\
\hline 546.4359 & 546.5066 & 13.781 & 0.009 & 47 & SHU & $R_{\mathrm{C}}$ \\
\hline 546.4375 & 546.4540 & 14.009 & 0.008 & 2 & SHU & $C V$ \\
\hline 546.4691 & 546.4796 & 14.396 & 0.013 & 14 & JSJ & $B$ \\
\hline 546.6157 & 546.7485 & 13.847 & 0.004 & 242 & $\mathrm{LCO}$ & $C V$ \\
\hline 546.6840 & 546.9420 & 14.179 & 0.017 & 270 & SRI & $C V$ \\
\hline 547.2813 & 547.4122 & 2.134 & 0.025 & 31 & CRI & $R_{\mathrm{C}}$ \\
\hline 547.2853 & 547.4100 & 1.592 & 0.019 & 31 & CRI & $B$ \\
\hline 547.2940 & 547.4115 & 1.980 & 0.023 & 30 & CRI & $V$ \\
\hline 547.2953 & 547.4128 & 2.229 & 0.025 & 30 & CRI & $I_{\mathrm{C}}$ \\
\hline 547.3109 & 547.5321 & 2.162 & 0.013 & 500 & CRI & $C V$ \\
\hline 547.3536 & 547.4979 & 14.165 & 0.028 & 134 & SHU & $R_{\mathrm{C}}$ \\
\hline 547.3596 & 547.4988 & 14.220 & 0.019 & 6 & SHU & $C V$ \\
\hline 547.4130 & 547.6069 & 14.341 & 0.017 & 351 & IMi & $V$ \\
\hline 547.4426 & 547.5714 & 14.179 & 0.005 & 181 & Trt & $V$ \\
\hline 547.5081 & 547.6157 & 14.139 & 0.007 & 125 & BSM & $C V$ \\
\hline 547.6819 & 547.9724 & 14.355 & 0.012 & 648 & SGE & $C V$ \\
\hline 548.2983 & 548.5226 & 2.155 & 0.056 & 52 & CRI & $B$ \\
\hline 548.2998 & 548.5200 & 2.439 & 0.040 & 52 & CRI & $V$ \\
\hline
\end{tabular}


Table E2. Log of observations of the 2016 outburst of 1SWASP J1621 (continued).

\begin{tabular}{rrrrrcc}
\hline Start* & End $^{*}$ & Mag $^{\dagger}$ & Error $^{\ddagger}$ & $N^{\S}$ & Obs & Band $^{\#}$ \\
\hline 548.3005 & 548.5207 & 2.526 & 0.032 & 52 & CRI & $R_{\mathrm{C}}$ \\
548.3011 & 548.5213 & 2.557 & 0.025 & 52 & CRI & $I_{\mathrm{C}}$ \\
548.3439 & 548.4874 & 14.802 & 0.027 & 93 & SHU, NKa & $R_{\mathrm{C}}$ \\
548.3584 & 548.5426 & 2.473 & 0.012 & 416 & CRI & $C V$ \\
548.3680 & 548.4865 & 14.411 & 0.043 & 4 & SHU & $C V$ \\
548.3890 & 548.6118 & 14.598 & 0.014 & 266 & BPO & $C V$ \\
548.4251 & 548.5936 & 14.716 & 0.016 & 314 & IMi & $V$ \\
548.5078 & 548.6157 & 14.456 & 0.010 & 119 & BSM & $C V$ \\
548.6831 & 548.9865 & 14.714 & 0.012 & 541 & SGE & $C V$ \\
549.3060 & 549.4802 & 2.406 & 0.016 & 44 & CRI & $B$ \\
549.3074 & 549.4776 & 2.668 & 0.016 & 43 & CRI & $V$ \\
549.3082 & 549.4783 & 2.728 & 0.017 & 43 & CRI & $R_{\mathrm{C}}$ \\
549.3087 & 549.4789 & 2.699 & 0.017 & 43 & CRI & $I_{\mathrm{C}}$ \\
549.6008 & 549.8552 & 15.795 & 0.031 & 95 & CMJ & $B$ \\
549.6020 & 549.8564 & 15.010 & 0.020 & 96 & CMJ & $V$ \\
549.6277 & 549.8538 & 14.966 & 0.014 & 176 & RIT & $C V$ \\
549.6900 & 549.9682 & 14.985 & 0.010 & 548 & SGE & $C V$ \\
549.6976 & 549.9180 & 15.741 & 0.038 & 89 & GFB & $B$ \\
549.6985 & 549.9190 & 14.985 & 0.023 & 90 & GFB & $V$ \\
550.0408 & 550.1700 & 14.838 & 0.010 & 338 & OKU & $C V$ \\
550.0501 & 550.2506 & 14.832 & 0.012 & 308 & Kis & $C V$ \\
550.0555 & 550.2780 & 14.625 & 0.010 & 284 & Ioh & $R_{\mathrm{C}}$ \\
550.2851 & 550.5242 & 2.734 & 0.030 & 60 & CRI & $B$ \\
550.2866 & 550.5216 & 2.921 & 0.023 & 59 & CRI & $V$ \\
550.2873 & 550.5223 & 2.936 & 0.021 & 59 & CRI & $R_{\mathrm{C}}$ \\
550.2879 & 550.5229 & 2.860 & 0.018 & 59 & CRI & $I_{\mathrm{C}}$ \\
550.3179 & 550.6242 & 14.940 & 0.012 & 183 & BSM & $C V$ \\
550.3451 & 550.5412 & 15.016 & 0.082 & 6 & SHU & $C V$ \\
550.6839 & 550.9838 & 15.127 & 0.009 & 541 & SGE & $C V$ \\
550.6942 & 550.9266 & 15.103 & 0.015 & 136 & SRI & $C V$ \\
550.6974 & 550.9731 & 15.972 & 0.020 & 230 & GFB & $B$ \\
551.2732 & 551.5204 & 2.999 & 0.028 & 62 & CRI & $B$ \\
551.2747 & 551.5218 & 3.096 & 0.025 & 62 & CRI & $V$ \\
551.2754 & 551.5226 & 3.061 & 0.022 & 62 & CRI & $R_{\mathrm{C}}$ \\
551.2760 & 551.5232 & 2.963 & 0.019 & 62 & CRI & $I_{\mathrm{C}}$ \\
552.4002 & 552.4594 & 15.087 & 0.021 & 32 & SHU, Nka & $C V$ \\
552.6653 & 552.8563 & 16.368 & 0.024 & 72 & CMJ & $B$ \\
\hline & & & & & & \\
\hline 50.04
\end{tabular}


Table E2. Log of observations of the 2016 outburst of 1SWASP J1621 (continued).

\begin{tabular}{rrrrrcc}
\hline Start $^{*}$ & End $^{*}$ & Mag $^{\dagger}$ & Error $^{\ddagger}$ & $N^{\S}$ & Obs & Band $^{\#}$ \\
\hline 552.6666 & 552.8550 & 15.385 & 0.023 & 70 & CMJ & $V$ \\
553.5893 & 553.8566 & 16.317 & 0.024 & 103 & CMJ & $B$ \\
553.5905 & 553.8553 & 15.325 & 0.017 & 102 & CMJ & $V$ \\
553.6977 & 553.9739 & 16.306 & 0.014 & 230 & GFB & $B$ \\
553.7135 & 553.8156 & 15.181 & 0.012 & 26 & SGE & $V$ \\
554.0120 & 554.1428 & 15.103 & 0.007 & 289 & OKU & $C V$ \\
554.3890 & 554.5240 & 15.312 & 0.018 & 71 & PVE & $V$ \\
554.4558 & 554.5183 & 15.092 & 0.012 & 67 & SHU, Nka & $C V$ \\
554.6905 & 554.9057 & 15.333 & 0.024 & 72 & SGE & $V$ \\
554.6935 & 554.9087 & 16.363 & 0.036 & 72 & SGE & $B$ \\
555.3449 & 555.5094 & 14.169 & 0.066 & 9 & PVE & $I_{\mathrm{C}}$ \\
555.3524 & 555.5439 & 15.359 & 0.025 & 55 & PVE & $V$ \\
555.3917 & 555.5158 & 16.326 & 0.085 & 8 & PVE & $B$ \\
555.6884 & 555.9814 & 15.211 & 0.023 & 49 & SGE & $V$ \\
555.6913 & 555.9845 & 16.242 & 0.028 & 53 & SGE & $B$ \\
556.3195 & 556.5463 & 14.141 & 0.019 & 61 & PVE & $I_{\mathrm{C}}$ \\
556.3574 & 556.5290 & 16.347 & 0.085 & 8 & PVE & $B$ \\
556.3631 & 556.5347 & 15.311 & 0.050 & 8 & PVE & $V$ \\
556.6001 & 556.8552 & 16.352 & 0.021 & 99 & CMJ & $B$ \\
556.6014 & 556.8539 & 15.352 & 0.018 & 97 & CMJ & $V$ \\
556.6899 & 556.9798 & 15.297 & 0.020 & 94 & SGE & $V$ \\
556.6930 & 556.9829 & 16.310 & 0.026 & 94 & SGE & $B$ \\
557.3278 & 557.4053 & 14.093 & 0.034 & 14 & PVE & $I_{\mathrm{C}}$ \\
557.3304 & 557.4133 & 15.278 & 0.045 & 15 & PVE & $V$ \\
558.3199 & 558.5466 & 15.373 & 0.019 & 99 & PVE & $V$ \\
563.0314 & 563.0755 & 14.638 & 0.010 & 62 & OKU & $R_{\mathrm{C}}$ \\
566.0187 & 566.1714 & 14.774 & 0.013 & 160 & OKU & $R_{\mathrm{C}}$ \\
617.3656 & 617.5738 & 15.229 & 0.009 & 288 & RPc & $C V$ \\
630.3350 & 630.5050 & 15.238 & 0.009 & 236 & RPc & $C V$ \\
665.2928 & 665.3731 & 15.202 & 0.012 & 106 & RPc & $C V$ \\
823.4359 & 823.5977 & 14.696 & 0.143 & 30 & WTH & $R_{\mathrm{C}}$ \\
\hline & & & &
\end{tabular}

*BJD-2457000.0.

${ }^{\dagger}$ Mean magnitude. Here, CRI reports the relative magnitude.

${ }^{\ddagger} 1 \sigma$ of mean magnitude.

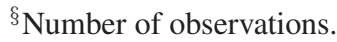

"Observer's code: GFB (William Goff), Kis (Seiichiro Kiyota), COO (Lewis M. Cook), Ioh (Hiroshi Itoh), CRI (Crimean Observatory), OKU (Osaka Kyoiku Univ. team), Trt (Tamás Tordai), RPc (Roger D. Pickard), IMi (Ian Miller), RIT (Michael Richmond), SGE (Geoff Stone), SHU (S. Shugarov team), SRI (Richard Sabo), LCO (Colin Littlefield), NKa (Natalia Katysheva \& Sergei Yu. Shugarov), PVE (Velimir Popov), HBB (Barbara Harris), CMJ (Michael Cook), WTH (Wikander, Thomas), Mic (Raúl Michel), BSM (Stephen M. Brincat), JSJ (Steve Johnson).

"Filter. " $C V$ " means no (clear) filter. 
Table E3. Log of observations of the 2006 outburst and the 2013 quiescence of BD Pav.

\begin{tabular}{rrrrrrr}
\hline Start $^{*}$ & End $^{*}$ & Mag $^{\dagger}$ & Error $^{\ddagger}$ & $N^{\S}$ & Obs & Band $^{\#}$ \\
\hline 979.2944 & 79.4928 & 12.481 & 0.003 & 539 & MLF & $C V$ \\
980.1966 & 80.5440 & 12.540 & 0.002 & 903 & MLF & $C V$ \\
982.1992 & 82.5138 & 12.635 & 0.003 & 851 & MLF & $C V$ \\
983.2130 & 83.5227 & 12.699 & 0.003 & 880 & MLF & $C V$ \\
984.1914 & 84.5195 & 12.776 & 0.003 & 930 & MLF & $C V$ \\
985.1892 & 85.5080 & 12.965 & 0.003 & 858 & MLF & $C V$ \\
3454.7143 & 2554.8467 & 15.152 & 0.114 & 150 & OAR & $V$ \\
3455.6887 & 2555.9171 & 15.157 & 0.144 & 220 & OAR & $V$ \\
3457.7425 & 2557.9143 & 15.101 & 0.109 & 167 & OAR & $V$ \\
3460.7053 & 2560.8474 & 15.039 & 0.030 & 9 & OAR & $V$ \\
3461.7647 & 2561.7685 & 14.956 & 0.015 & 5 & OAR & $V$ \\
3462.7649 & 2562.7688 & 15.077 & 0.033 & 5 & OAR & $V$ \\
3463.7621 & 2563.7657 & 15.156 & 0.016 & 5 & OAR & $V$ \\
\hline
\end{tabular}

*BJD-2453000.0.

${ }^{\dagger}$ Mean magnitude.

${ }^{\ddagger} 1 \sigma$ of mean magnitude.

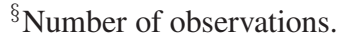

"Observer's code: MLF (Berto Monard).

${ }^{\#}$ Filter. " $C V$ " means no (clear) filter. 
Table E4. Log of observations of the 2009 outburst of V364 Lib.

\begin{tabular}{|c|c|c|c|c|c|c|}
\hline Start* & End ${ }^{*}$ & $\operatorname{Mag}^{\dagger}$ & Error $^{\ddagger}$ & $N^{\S}$ & $\mathrm{Obs}^{\|}$ & Band $\#$ \\
\hline 928.0757 & 28.2011 & 10.412 & 0.001 & 405 & Mhh & $\bar{V}$ \\
\hline 928.1202 & 28.2177 & 11.478 & 0.003 & 223 & $\mathrm{Njh}$ & $C V$ \\
\hline 928.2010 & 28.2883 & 1.822 & 0.001 & 481 & OUS & $C V$ \\
\hline 928.2017 & 28.2036 & 10.569 & 0.006 & 6 & Mhh & $B$ \\
\hline 929.1080 & 29.1733 & 10.711 & 0.001 & 89 & $\mathrm{Kis}$ & $C V$ \\
\hline 929.1197 & 29.2284 & 1.893 & 0.002 & 351 & OUS & $C V$ \\
\hline 930.0970 & 30.2722 & 11.460 & 0.001 & 412 & $\mathrm{Njh}$ & $C V$ \\
\hline 930.1155 & 30.2758 & 1.880 & 0.001 & 885 & OUS & $C V$ \\
\hline 930.1287 & 30.1968 & 10.732 & 0.002 & 96 & $\mathrm{Kis}$ & $C V$ \\
\hline 930.1431 & 30.2359 & 10.436 & 0.002 & 138 & Mhh & V \\
\hline 930.1434 & 30.2355 & 10.628 & 0.002 & 136 & Mhh & $B$ \\
\hline 931.0911 & 31.3198 & 11.493 & 0.001 & 537 & $\mathrm{Njh}$ & $C V$ \\
\hline 931.0944 & 31.1718 & 10.710 & 0.003 & 104 & $\mathrm{Kis}$ & $C V$ \\
\hline 932.0938 & 32.2945 & 11.552 & 0.001 & 465 & $\mathrm{Njh}$ & $C V$ \\
\hline 932.1091 & 32.1847 & 10.770 & 0.003 & 102 & $\mathrm{Kis}$ & $C V$ \\
\hline 932.1812 & 32.2890 & 10.729 & 0.001 & 410 & Mhh & $B$ \\
\hline 937.1064 & 37.1435 & 11.750 & 0.002 & 88 & $\mathrm{Njh}$ & $C V$ \\
\hline 938.0842 & 38.2074 & 11.906 & 0.003 & 237 & $\mathrm{Njh}$ & $C V$ \\
\hline 939.2176 & 39.3233 & 11.199 & 0.001 & 591 & Mhh & $C V$ \\
\hline 945.1195 & 45.2613 & 12.179 & 0.001 & 335 & $\mathrm{Njh}$ & $C V$ \\
\hline 947.1338 & 47.1456 & 12.247 & 0.005 & 29 & $\mathrm{Njh}$ & $C V$ \\
\hline 949.0912 & 49.2735 & 12.347 & 0.002 & 422 & $\mathrm{Njh}$ & $C V$ \\
\hline 950.1414 & 50.2637 & 12.363 & 0.002 & 288 & $\mathrm{Njh}$ & $C V$ \\
\hline 951.0445 & 51.2736 & 12.407 & 0.002 & 540 & $\mathrm{Njh}$ & $C V$ \\
\hline 951.3060 & 51.6199 & 0.042 & 0.001 & 1623 & MLF & $V$ \\
\hline 952.0562 & 52.1361 & 12.431 & 0.002 & 189 & $\mathrm{Njh}$ & $C V$ \\
\hline 953.0907 & 53.2577 & 12.433 & 0.001 & 392 & $\mathrm{Njh}$ & $C V$ \\
\hline 965.0846 & 65.2432 & 12.440 & 0.001 & 370 & $\mathrm{Njh}$ & $C V$ \\
\hline
\end{tabular}

*BJD-2454000.0.

${ }^{\dagger}$ Mean magnitude. Here, OUS reports the relative magnitude.

${ }^{\ddagger} 1 \sigma$ of mean magnitude.

$\S$ Number of observations.

"Observer's code: Mhh (Hiroyuki Maehara), Njh (Kazuhiro Nakajima), OUS (Okayama U. of Science), Kis (Seiichiro Kiyota).

${ }^{\#}$ Filter. " $C V$ " means no (clear) filter. 
Table E5. Log of spectroscopic observations of V364 Lib.

\begin{tabular}{|c|c|c|c|c|c|c|c|}
\hline Date $^{*}$ & $\mathrm{Name}^{\dagger}$ & Start Time ${ }^{\ddagger}$ & End Time ${ }^{\S}$ & $\operatorname{Exp}^{\|}$ & Number $\#$ & Grating & Site \\
\hline 2009-04-07 & V364 Lib & 54929.1854 & 54929.2062 & 60 & 11 & Grism & $\mathrm{G}$ \\
\hline 2009-04-09 & V364 Lib & 54931.1862 & 54931.2022 & 60 & 15 & Grism & G \\
\hline 2009-04-10 & V364 Lib & 54932.1536 & 54932.1703 & 60 & 12 & Grism & G \\
\hline- & V364 Lib & 54932.1953 & 54932.2043 & 60 & 9 & Grism & $\mathrm{G}$ \\
\hline 2009-04-15 & V364 Lib & 54937.0754 & 54937.0764 & 30 & 3 & $I_{\mathrm{C}}$ & G \\
\hline- & V364 Lib & 54937.0816 & 54937.0948 & 60 & 11 & Grism & G \\
\hline 2009-04-18 & V364 Lib & 54940.1137 & 54940.1151 & 60 & 2 & Grism & G \\
\hline 2009-04-19 & V364 Lib & 54941.0797 & 54941.0901 & 60 & 10 & Grism & G \\
\hline $2009-04-22$ & V364 Lib & 54944.0798 & 54944.0902 & 60 & 10 & Grism & G \\
\hline $2009-04-23$ & V364 Lib & 54945.1173 & 5494.1257 & 60 & 5 & Grism & G \\
\hline $2009-04-26$ & V364 Lib & 54948.1084 & 54948.1209 & 120 & 5 & Grism & G \\
\hline 2009-04-27 & V364 Lib & 54949.0751 & 54949.0820 & 60 & 6 & Grism & G \\
\hline 2009-04-28 & V364 Lib & 54950.1300 & 54950.1473 & 60 & 12 & Grism & G \\
\hline $2009-05-02$ & V364 Lib & 54954.0627 & 54954.0738 & 60 & 10 & Grism & G \\
\hline $2009-05-05$ & V364 Lib & 54961.8932 & 54961.9392 & 900 & 5 & std-Yd & S \\
\hline - & V364 Lib & 54961.9619 & 54961.0183 & 900 & 6 & std-Bc & S \\
\hline- & V364 Lib & 54962.0348 & 54962.0573 & 900 & 3 & std-Yd & S \\
\hline 2009-05-08 & V364 Lib & 54960.1634 & 54960.0759 & 60 & 10 & Grism & G \\
\hline 2009-05-13 & V364 Lib & 54965.1412 & 54965.0419 & 20 & 3 & $\mathrm{R}$ & G \\
\hline- & V364 Lib & 54965.1510 & 54965.1579 & 60 & 7 & Grism & G \\
\hline $2009-05-20$ & V364 Lib & 54972.1113 & 54972.1183 & 60 & 6 & Grism & G \\
\hline
\end{tabular}

* Observational date (Japan Standard Time).

${ }^{\dagger}$ Name of star.

${ }^{\ddagger}$ Start time of observations in the unit of BJD-2400000.

${ }^{\S}$ End time of observations in the unit of BJD-2400000.

${ }^{\|}$Exposure time of each observation in unit of seconds.

\# Number of observations.

"Diffraction or echelle gratings.

** Name of observatory: S (Subaru Observatory), G (Gunma Observatory).

Table E6. Radial velocity measured in the 2009 quiescence of V364 Lib.

\begin{tabular}{rrrr}
\hline Time $^{*}$ & Phase $^{\dagger}$ & Radial Velocity & Error \\
\hline 54929.1934 & 0.7912 & -107.8 & 10.8 \\
54931.1895 & 0.6329 & -50.3 & 11.3 \\
54932.1952 & 0.0648 & 73.3 & 13.7 \\
54937.0838 & 0.0243 & 42.0 & 22.2 \\
54941.0801 & 0.7135 & -99.8 & 37.9 \\
54944.1177 & 0.0379 & -5.6 & 46.4 \\
54945.1577 & 0.5186 & -12.3 & 52.5 \\
\hline
\end{tabular}

${ }^{*}$ Time of observations in the unit of BJD-2400000.

${ }^{\dagger}$ Orbital phase under the assumption that the orbital period is $0.7024293 \mathrm{~d}$. 
Table E7. Radial velocity measured in the 2009 outburst of V364 Lib.

\begin{tabular}{rrrr}
\hline Time $^{*}$ & Phase $^{\dagger}$ & Radial Velocity & Error \\
\hline 54961.3873 & 0.3746 & -49.7 & 4.3 \\
54961.3994 & 0.3917 & -38.1 & 3.3 \\
54961.4107 & 0.4078 & -34.3 & 3.7 \\
54961.4220 & 0.4239 & -28.4 & 3.7 \\
54961.4334 & 0.4401 & -21.1 & 4.1 \\
54961.4561 & 0.4724 & -6.0 & 5.3 \\
54961.4676 & 0.4888 & 2.4 & 4.3 \\
54961.4788 & 0.5048 & 8.5 & 4.2 \\
54961.4901 & 0.5208 & 13.4 & 3.1 \\
54961.5013 & 0.5367 & 22.5 & 3.1 \\
54961.5125 & 0.5527 & 30.8 & 2.2 \\
54961.5289 & 0.5762 & 40.5 & 6.7 \\
54961.5403 & 0.5923 & 49.5 & 5.5 \\
54961.5515 & 0.6083 & 51.4 & 4.5 \\
\hline${ }^{*}$ Time of observations in the unit of BJD-2400000. \\
${ }^{\dagger}$ Orbital phase under the assumption that the orbital \\
period is 0.7024293 d.
\end{tabular}



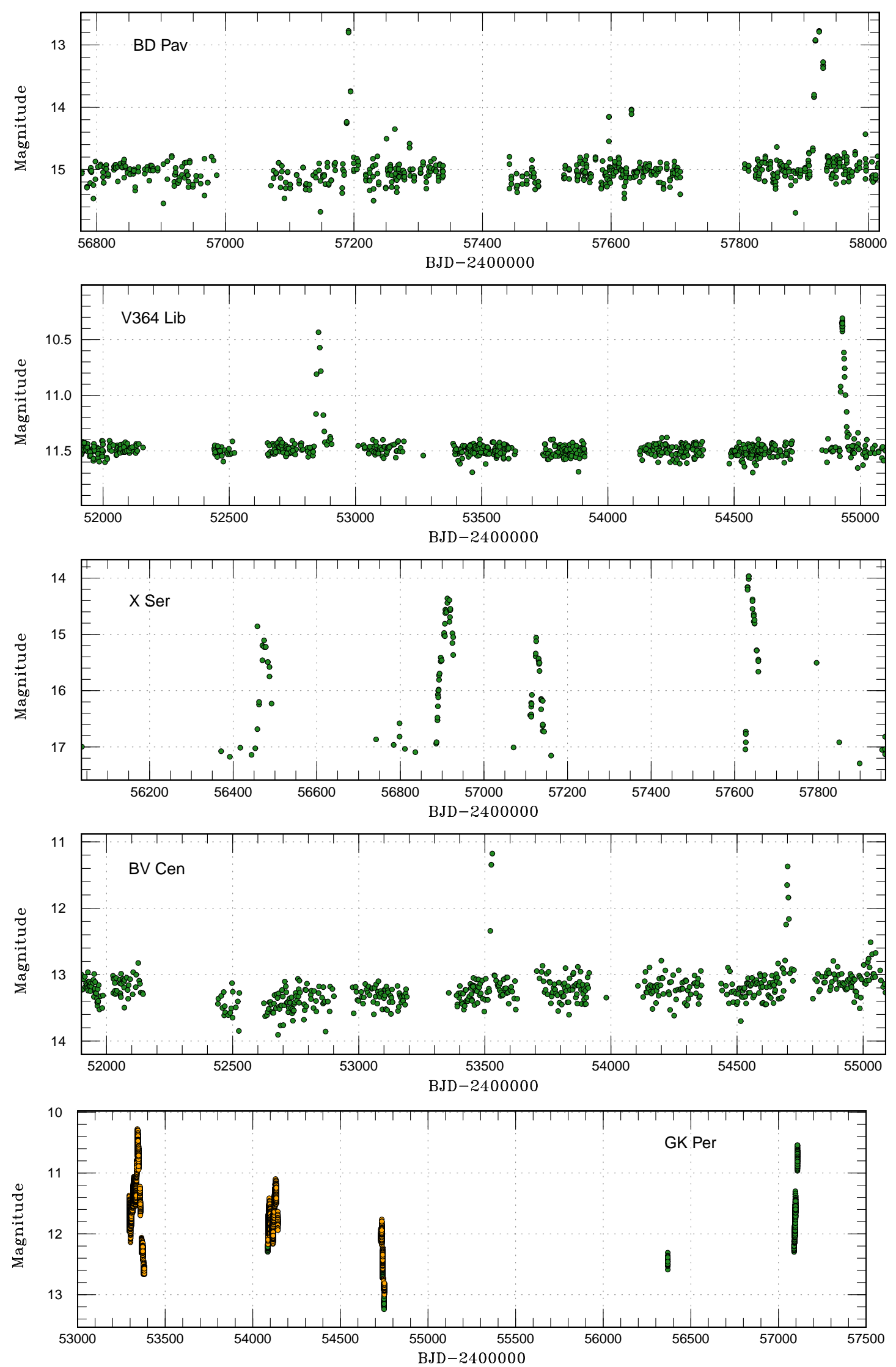

Fig. E1. Long-term light curves in the long-period objects discussed in this paper. The light curves of V364 Lib and BV Cen are derived from the ASAS-3 data archive. The light curves of BD Pav and X Ser are derived from the ASAS-SN data archive. Green and orange points represent the observations in the $V$ band and no (clear) filter, respectively. 